УДК 620.952

\title{
ТЕХНОЛОГИЧЕСКИЕ РЕШЕНИЯ ПО УТИЛИЗАЦИИ ЖИДКИХ ПРОДУКТОВ МЕДЛЕННОГО ПИРОЛИЗА ДРЕВЕСНОЙ БИОМАССЫ
}

\author{
Слюсарский Константин Витальевич1, \\ slyuskonst@gmail.com
}

\section{Ларионов Кирилл Борисович2,} laryk070@gmail.com

\author{
Ивашкина Елена Николаевна', \\ ivashkinaen@tpu.ru
}

\author{
Заворин Александр Сергеевич1, \\ zavorin@tpu.ru
}

\author{
Губин Владимир Евгеньевич1, \\ gubin@tpu.ru \\ 1 Национальный исследовательский Томский политехнический университет, \\ Россия, 634050, г. Томск, пр. Ленина, 30. \\ 2 Кузбасский государственный технический университет им. Т.Ф. Горбачева, \\ Россия, 650000, г. Кемерово, ул. Весенняя, 28.
}

\begin{abstract}
Актуальность работы обусловлена возрастающим интересом к технологиям пиролиза биомассы для снижения углеродного следа процессов её переработки. Для повышения экономической и энергетической эфффективности данных технологических решений необходимо максимально полное использование всех материальных и энергетических потоков, одним из которых является формииование жидких продуктов пиролиза (т. н. пиролизной жидкости, или бионефрти).

Цель: классификация и выделение технологических решений для утилизации жидких продуктов пиролиза древесной биомассы с определением уровня технической готовности.

Методы: аналитический обзор тематических публикаций с использованием материалов баз данных РИНЦ, Scopus u Web of Science, уровень технической готовности оценивался согласно шкале TRL.

Результаты. Идентифицированы, классифицированы и описаны основные методы утилизации пиролизной жидкости, получаемой в результате медленного пиролиза древесной биомассы. Представлен обзор технологических решений и научноисследовательских работ в области утилизации жидких продуктов медленного пиролиза. Наиболее распространенными технологиями преобразования пиролизной жидкости с получением тепловой и/или электрической энергии являются прямое сжигание в горелках котлов и теплогенераторов, в камере сгорания газотурбинных двигателей, газификация с получением синтез-газа, а также использование в качестве топлива поршневых двигателей. Технологии получения химических продуктов и веществ разделяются на простые (получение компонент дорожного строительства, консерванта для древесины, френолформальдегидных и омыленных смол и др.) и сложные, требующие комплексной многостадийной переработки. Установлено, что наибольшим уровнем технической готовности обладают технологии энергетического применения данного продукта, в то время как наибольшей экономической эфффективностью обладают технологии химического применения, несмотря на относительно низкий уровень готовности. Коммерческие решения по утилизации пиролизной жидкости доступны в области её сжигания в горелках и дизель-генераторах.
\end{abstract}

\section{Ключевые слова:}

Пиролиз, биомасса, сжигание, горение, бионефть, пиролизная жидкость, биоуголь.

\section{Введение}

Интеграция биомассы в ТЭК является одним из приоритетных направлений развития энергетической отрасли из-за экологичности данного вида топлива (биомасса является углероднонейтральным топливом [1]). Отходы лесопиления - один из самых распространенных видов биомассы, особенно в России. Известно, что пиролитическая обработка древесины является перспективной технологией для получения различных полезных продуктов в твердой, жидкой и газообразной фазой [2].

Существующие технологии пиролиза могут быть классифицированы по нескольким признакам: по составу используемой газовой среды (азот, водяной пар или диоксид углерода), по конструкции реактора (ре- акторы плотного, кипящего слоя) и т. п. Однако одним из наиболее значимых признаков, с точки зрения характеристик получаемых продуктов, является скорость нагрева. По скорости нагрева выделяют технологии флэш $\left(>1000{ }^{\circ} \mathrm{C} / \mathrm{c}\right)$, быстрого $\left(>10^{\circ} \mathrm{C} / \mathrm{c}\right)$ и медленного $\left(<1{ }^{\circ} \mathrm{C} / \mathrm{c}\right)$ пиролиза [2, 3]. Быстрый пиролиз характеризуется высоким выходом жидкой фракции (до 75 мас. \%), а медленный пиролиз приводит к более высокому содержанию в продуктах твердой и газовой фазы (до 40 и 35 мас. \% соответственно) [2, 4-6]. Для повышения конкурентоспособности таких технологий твердый продукт пиролиза можно использовать в качестве сорбента [5, 6], удобрений [7] или древесного угля $[4,8]$. Для увеличения доли получаемого твердофазного продукта обычно используют медлен- 
ный пиролиз. Несмотря на то, что основным продуктом медленного пиролиза является продукт в твердой фазе, в ходе его реализации образуется порядка 30 мас. \% жидких продуктов. Для повышения эффективности технологий пиролиза биомассы эти материальные потоки также должны максимально эффективно утилизироваться. Дополнительными достоинствами технологий медленного пиролиза является простота топливоподготовки и малые размеры реактора, что позволяет их использовать непосредственно на месте получения биомассы [9]. Это позволяет снизить затраты на транспортировку биомассы, что является важным фактором для обеспечения эффективности данных технологий [10]. Поэтому технологии утилизации жидких продуктов также должны быть достаточно простыми.

Технологии промышленного использования жидких продуктов именно быстрого пиролиза биомассы в настоящее время наиболее хорошо разработаны [11] ввиду более высокого выхода жидкофазных продуктов конверсии (до $75 \%$ от начальной массы). К особенностям данного метода относится крайне низкий выход продукта в твердой фазе [12], что ограничивает его применимость к технологиям получения биококса и его аналогов. Для производства биококса и его аналогов более подходящими являются методы, основанные на процессах пиролиза в условиях медленного нагрева (менее $10{ }^{\circ} \mathrm{C} /$ мин), позволяющие достигать более высокого выхода тведофазного продукта (до $40-45 \%$ от массы исходного сырья [13-15]). Несмотря на значительные отличия реализации непосредственно процесса, состав жидкофазных продуктов быстрого и медленного пиролиза достаточно близок. В общем случае пиролизная жидкость содержит органические соединения широкого спектра, среди которых наибольшая концентрация альдегидов, кетонов, моносахаридов и фенолов [16]. Помимо выделения отдельных компонент для дальнейшего использования [17], данная жидкость может использоваться в качестве топлива котельных установок [18] и камер сгорания различных установок $[19,20]$. Условно подобные технологии можно разделить на две группы: технологии энергетического применения и химической переработки.

Целью настоящей публикации является комплексная оценка технологических решений в области утилизации жидких углеводородов, полученных в результате медленного пиролиза древесных отходов. Аналитический обзор направлен на решения с учетом уровня их технологической готовности и опыта реализации, причем наибольшее внимание уделяется наиболее приближенным к практическому внедрению. Поскольку наиболее востребованным продуктом медленного пиролиза является углеродистый остаток, обзор посвящен технологическим решениям, соответствующим по уровню сложности технологиям пиролиза в данной области.

\section{Технологии энергетического применения} пиролизной жидкости

Процесс пиролиза сопряжен с высокими затратами теплоты на нагрев среды и компенсацию эндотерми- ческих реакций и процессов, таких как, например, испарение адсорбированных и полученных в ходе термодеструкции жидких компонентов. Поэтому большинство промышленных комплексов пиролиза древесного сырья используют энергию сгорания данных веществ для полной или частичной компенсации энергозатрат. Наиболее проработанными на данный момент решениями являются технологии применения пиролизной жидкости в качестве топлива котлоагрегатов $[18,21,22]$ и дизель-генераторов [23, 24]. Использование пиролизной жидкости в качестве топлива для газотурбинных и газификационных установок $[11,24,25]$ является технологическим решением с меньшей степенью проработки.

\section{Технологии прямого сжигания пиролизной жидкости}

Сжигание пиролизной жидкости с использованием стандартного энергетического оборудования усложняется существенным отличием ряда характеристик от классического жидкого топлива. В частности, такими особенностями называют относительно высокую кислотность и зольность, большую долю балласта в составе (в основном воды), меньшую теплотворную способность и необходимость поддерживать высокую полноту сгорания, т. к. продукты неполного сгорания некоторых компонент пиролизного масла могут быть токсичны [20].

Наибольший прогресс был достигнут применительно к установкам быстрого пиролиза топлива. Однако схожесть составов жидкостей, получаемых в результате быстрого и медленного пиролиза древесной биомассы, позволяет проецировать данный опыт на исследуемые условия [11]. Рядом компаний было реализовано промышленное внедрение комплексных установок по получению пиролизной жидкости и её использованию для целей производства теплоты для собственных нужд и внешних потребителей. К таким компаниям относятся Fortum (Финляндия) [26, 27], Ensyn Fuels (Канада) [28], Valmet (Финляндия) [29], Stork (Нидерланды) [30] и др. Так, например, Valmet предлагает комплексные решения по сжиганию бионефти для совместного производства тепловой и электрической энергии, а компания Stork предлагает готовые решения по сжиганию пиролизной жидкости для производства перегретого пара [30] (рис. 1).

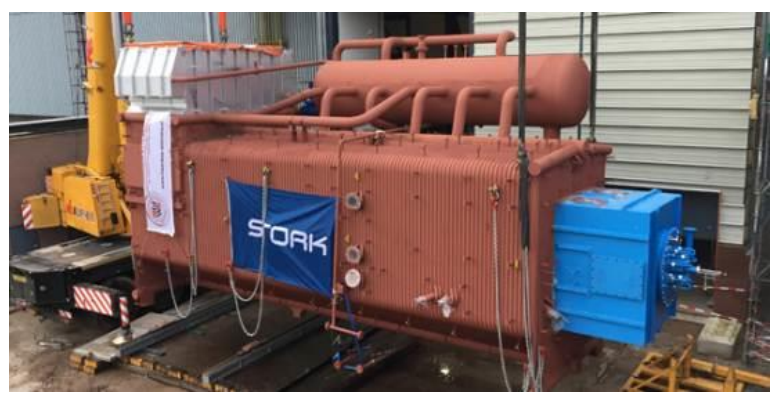

Pис. 1. Внешний вид парового котла фирмы Stork, использующего пиролизную жидкость в качестве топлива [30]

Fig. 1. Image of steam boiler by Stork [30] which uses pyrolysis liquid as fuel 
В России зрелые технологические решения в данной сфере в настоящее время либо отсутствуют, либо находятся на ранних стадиях научно-технических исследований и апробации $[24,31,32]$. При этом, как было отмечено ранее, схожесть теплотворной способности классического жидкого топлива с теплотой сгорания пиролизной жидкости позволяет использовать существующие горелочные устройства. Например, в [22, 32] представлены результаты успешной апробации универсальной горелки «Kroll $\mathrm{GmbH»}$ KG/UB 55 при работе на пиролизной жидкости, полученной в ходе быстрого пиролиза древесины. Было установлено, что стабильное зажигание и горение топлива достигается при незначительном предварительном нагреве - менее $80^{\circ} \mathrm{C}$ - и увеличении мощности устройства электрического розжига (или использование дополнительной «подсветки» классическим топливом). Полученные данные хорошо согласуются с результатами аналогичных исследований за рубежом [20]. При этом во всех работах отмечается, что использование пиролизной жидкости позволяет улучшить экологические характеристики процесса горения - отмечается снижение выбросов оксидов азота. В [32] указано, что на базе ООО «Энергетика» (Казань, Россия) были проведены испытания котла мощностью 80 кВт при сжигании бионефти. При использовании испарительных горелок наблюдалось протекание вторичных реакций пиролиза с образованием пека смол (коксование поверхностей).

Основные рекомендации по модернизации стандартных горелочных устройств для использования пиролизной жидкости: должны использоваться коррозионностойкие материалы для форсунок, предварительный подогрев топлива должен быть ограничен, рекомендовано использование дополнительного устройства для розжига, а также необходим строгий контроль высокого уровня полноты сгорания топлива.

Использование пиролизной жидкости в качестве топлива для камер сгорания газовых турбин является гораздо менее проработанным техническим решением. Несмотря на схожие с котельными установками принципы эксплуатации, ввиду более суровых (в сравнении с горелками котельных установок) условий эксплуатации (высокие температуры и давление) указанные ранее недостатки играют более важную роль. Причем негативное воздействие распространяется не только на материалы непосредственно камеры сгорания, но и на расположенное за нею оборудование активные и сопловые лопатки газовой турбины. Ввиду высокой стоимости последней негативное влияние на данные элементы должно быть минимизировано.

Однако возможность присутствия твердых частиц в пиролизной жидкости и относительно высокая зольность могут сделать её использование в газотурбинной установке затруднительным. Между тем, одной из немногих опубликованных работ с описанием опыта промышленной эксплуатации подобной системы является [33]. Образец турбины ОГТ2500 мощностью 2,5 МВт был модифицирован сотрудниками корпорации Orenda Aerospace Corporation (Канада) для работы на биотопливе различного происхождения (рис. 2).

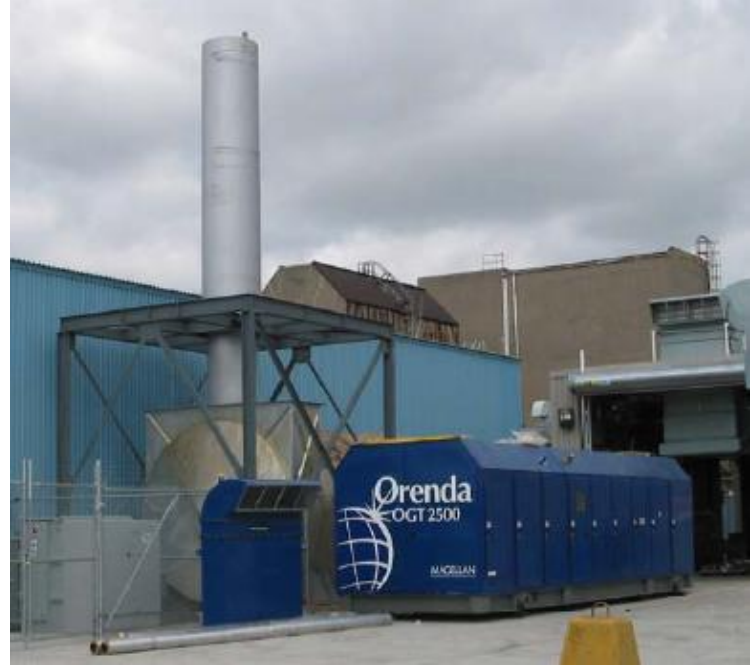

Puc. 2. Внешний вид установки ОГТ2500 [33]

Fig. 2. Image of the OGT2500 unit [33]

Указано, что существующая газотурбинная установка была модифицирована для работы на биотопливе. Была модифицирована камера сгорания, установлена система горячей очистки и дополнительной обработки топлива. При этом последние опубликованные данные по работе данной установки датируются 2005 г., и, по представленной в [11] информации, после модификации установка практически не эксплуатировалась. В [11] также упоминаются газотурбинные установки фирмы OPRA Turbines (Финляндия). На сайте корпорации [34] приведено описание газовой турбины ОР-16, способной, по заверениям производителя, работать на различном топливе, в том числе и пиролизной жидкости. В материалах конференций последних лет описывается успешный опыт демонстрационной эксплуатации газотурбинных двигателей OPRA OP16 при работе на бионефти $[35,36]$, однако данные по опыту промышленной эксплуатации подобных установок в литературе не представлены.

Отечественные разработки в данной сфере находятся на уровне научно-исследовательских работ [32]. В [37] представлены результаты теоретических и экспериментальных исследований (преимущественно, зарубежных) при работе камер сгорания с использованием жидких биотоплив различного происхождения - одной марки бионефти, полученной в ходе пиролиза автомобильных покрышек, и двух марок, полученных в ходе переработки двух разновидностей древесины. На основе представленных данных и опыта промышленной эксплуатации были сформированы характерные рекомендации по применению, схожие с рекомендациями, сформированными для горелочных устройств [37]. Помимо них, были выявлены следующие дополнительные требования: необходимость отсутствия твердых частиц большого размера в топливе, необходимость более строго контроля значений $\mathrm{pH}$ топлива и, при необходимости, влияния на данное значение путем внесения кислотных или щелочных добавок, более строгий контроль за содержанием серы в топливе для контроля и предотвращения образо- 
вания отложений (рис. 3) и высокотемпературной коррозии.

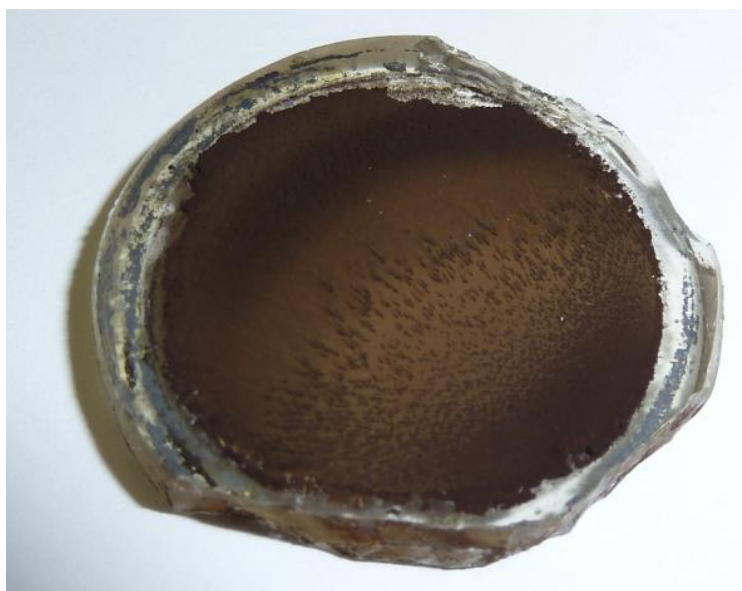

Puc. 3. Внешний вид отложений в камере сгорания газовой турбины при работе на жидких продуктах пиролиза древесины без контроля содержания серы [37]

Fig. 3. Image of deposits into gas turbine combustion chamber after using liquid products of wood pyrolysis as fuel without sulfur control [37]

Применение пиролизной жидкости в качестве топлива дизель-генераторов исследовано в зарубежной литературе достаточно детально. Наиболее полный обзор подобных научно-исследовательских работ представлен в [38]. Обширные опытнопромышленные испытания проводились с участием VTT (Финляндия) [39] и Wärtsilä (Финляндия) [40]. Все указанные ранее недостатки (кислотность, низкая теплота сгорания, высокая зольная балластность, возможность присутствия твердых частиц) сказывались и на особенностях эксплуатации дизельгенератора, в частности, на явлении ускоренного износа форсуночных игл (рис. 4). Установлено, что использование пиролизной жидкости позволяет достигать предельно высоких значений термического КПД до $45 \%$ - и значительно снизить выбросы оксидов азота, а также увеличить скорость горения топлива и уменьшить температурные градиенты в цилиндрах за счет высокой теплопроводности водяного пара.

Дополнительные требования к эксплуатации дизель-генераторов были сформулированы следующим образом.

1. Вся линия топливоснабжения (баки, фильтры, трубопроводы, форсунки и пр.) должна быть выполнена из коррозионностойких материалов для снижения негативного воздействия низкого $\mathrm{pH}$ топлива.

2. Необходимость организации контроля температуры и постоянного перемешивания пиролизной жидкости в баке для предотвращения её расслоения.

3. Необходимость строго контроля теплотворной способности топлива и отсутствия твердых частиц. Нерешенной остаётся проблема износа форсунок и интенсивного образования лакового нагара в двигате- ле. Возможными решениями могут стать использование добавок, предотвращающих данный процесс, или выборочное удаление элементов, имеющих наибольший вклад в процесс лакообразования. Также значительной проблемой является высокая вязкость подобного топлива, что усугубляется невозможностью значительного подогрева (выше $80^{\circ} \mathrm{C}$ ) для снижения вязкости. Содержание спиртов в жидкости также приводит к ухудшению его низкотемпературных свойств.

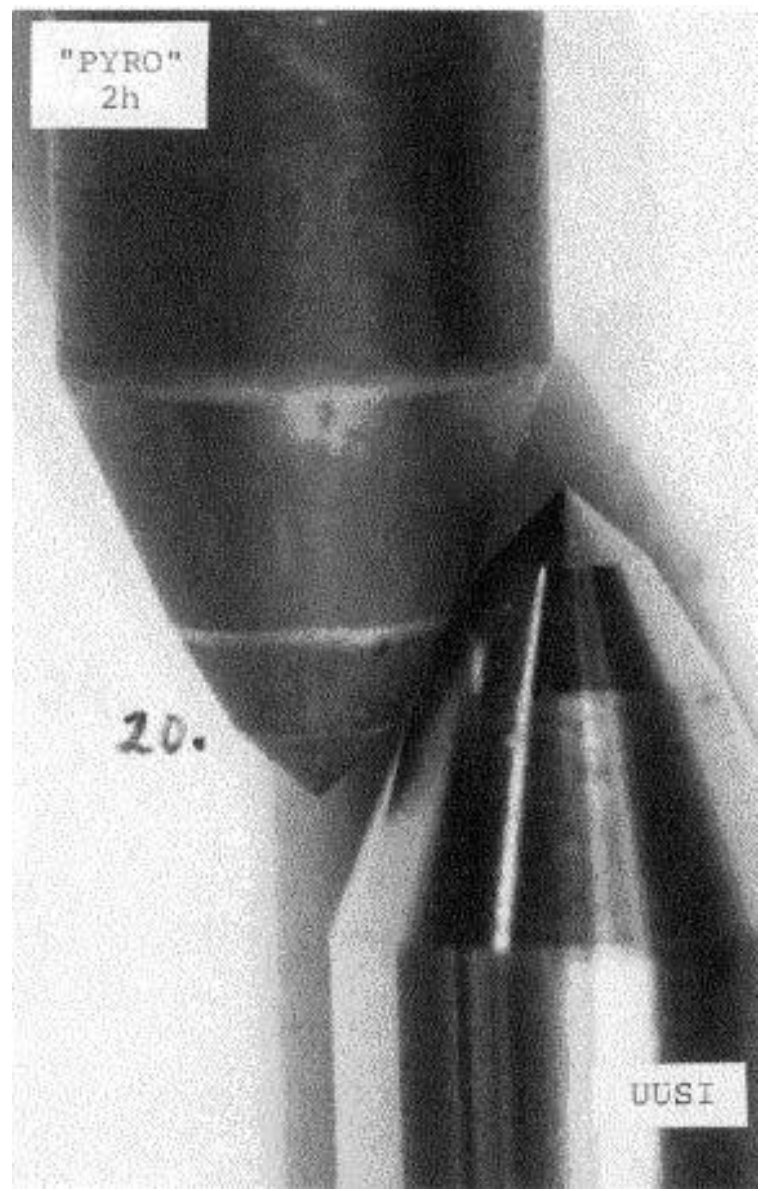

Pис. 4. Внешний вид форсуночных игл: новой (снизу) и после двух часов эксплуатаиии (сверху) [41]

Fig. 4. Image of the atomizing needle: new (at the bottom) and after 2 hof operation (at the top) [41]

Во многих работах $[11,23,41]$ отмечается необходимость использования различных вспомогательных топлив и присадок для обеспечения стабильного зажигания и горения исследованных составов. Наиболее перспективной добавкой к пиролизной жидкости является бутанол [42-44], позволяющий не только улучшить энергетические характеристики топлива, но и предотвратить его расслаивание и снизить вязкостные характеристики. Так, например, в [45] указано, что добавка 15 мас. \% бутанола позволяет достигать удовлетворительных характеристик работы дизельного двигателя, сопоставимых с параметрами, наблюдаемыми при работе на обычном дизельном топливе. Помимо бутанола и различных цетановых присадок, предлагается использование различных веществ, та- 
ких как этанол [46] или непосредственно дизель [23], что приводит к улучшению эксплуатационных характеристик, хотя и не столь существенному.

В отечественной литературе подобные работы практически не представлены. Имеется ограниченное число работ по применению схожих веществ в качестве топлива дизель-генераторов [47].

Wärtsilä в России предлагает поставку дизельгенераторов, способных работать на топливе различного происхождения, в т. ч. и из отходов различного происхождения. Предприятие BTG Biomass Technology Group (Нидерланды) [48] имеет опыт модификации существующих установок для работы на пиролизной жидкости [11]. При этом нередко требовался достаточно большой объем модификаций, целесообразность которых должна оцениваться в каждом конкретном случае.

Следует отметить, что в данном разделе не рассматривались системы, работающие на синтез-газе, полученном в ходе газификации/окисления пиролизной жидкости.

\section{Технологии газификации пиролизной жидкости}

Газификация пиролизной жидкости - процесс преобразования пиролизной жидкости в горючий газ за счет реакций неполного окисления углеродсодержащих компонент воздухом и/или водяным паром. Технологии использования газообразного топлива различного состава разработаны достаточно хорошо благодаря простоте обращения и универсальности соответствующих подходов [2, 49-52] - большинство оборудования, спроектированного для работы на классическом газообразном топливе, может использоваться при работе на газе различного состава с минимальным количеством модификаций. Большой спектр камер сгорания, поршневых двигателей и газотурбинных установок поставляются адаптирован- ными для работы в подобных условиях. Существует три принципиально отличающихся подхода к организации непосредственно процесса газификации $[52,53]$ : газификация в плотном слое, газификация в кипящем слое, газификация в потоке.

Применительно к жидкому топливу пригоден только последний способ - газификация пиролизной жидкости в потоке. В большинстве случаев такая газификация реализуется совместно с процессом пиролиза исходной древесины. Как правило, для этого достаточно поддерживать высокую температуру выделяющегося пиролизного газа во всем тракте. В таком случае могут применяться все указанные выше подходы. Однако такое решение приводит к максимально высокой доле балласта в получаемых продуктах, т. к. при частичной конденсации продуктов значительная часть влаги может быть отведена. Учитывая, что сконденсированная влага содержит фенол в значительной концентрации, иногда такой подход оправдан.

В зарубежной литературе представлено большое число исследований различной степени разработки и масштаба (рис. 5). В последнее время превалирует мнение о необходимости обеспечения максимальной компактности подобных установок ввиду низкой энергетической плотности биотоплива и большой стоимости его транспортировки, особенно на большие расстояния, поэтому большинство современных установок имеют невысокую мощность.

При этом имеющиеся промышленные образцы технологий газификации биомассы (например, технологии Siemens $[56,57])$ не выделяют элементов конденсации жидких продуктов реакции (рис. 6). Примечательно, что такие установки основаны на принципиально отличающемся от пиролиза подходе к переработке биомассы.

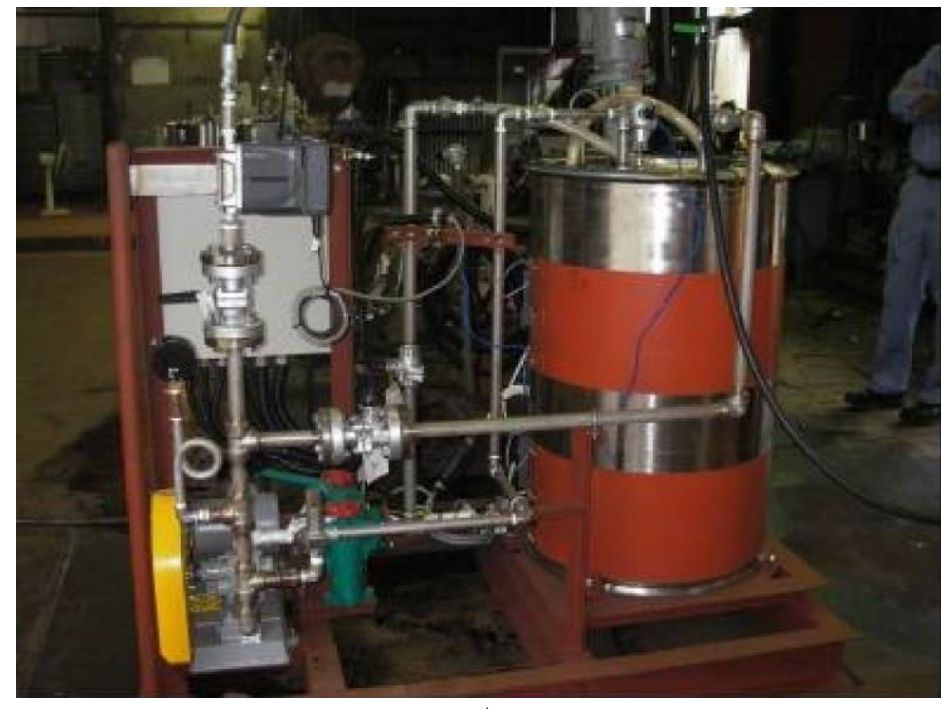

$a / a$

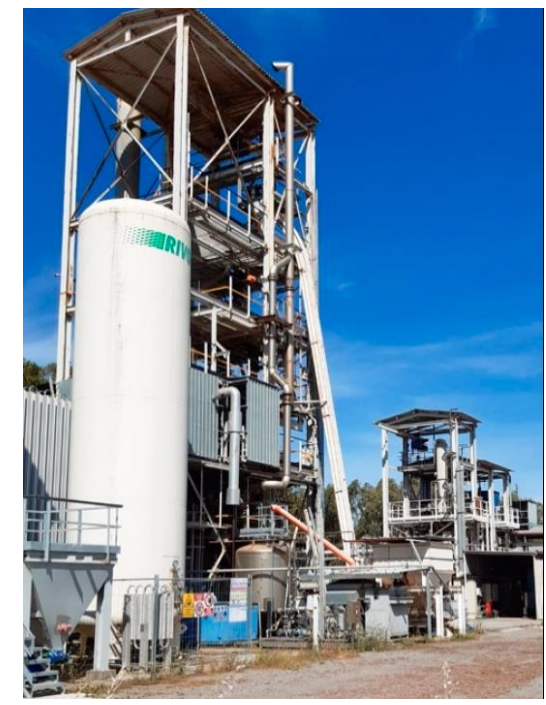

$\sigma / b$

Рис. 5. Опытно-промышленные установки газификачии: а) часть стенда газификации биомассы мощностью $2 \mathrm{MBm}$ в г. Сайтама, Япония [54]; б) опытно-промышленный стенд комплексной слоевой газификации мощуностью 5 МВт в г. Сотакарбо, Италия [55]

Fig. 5. Pilot-scale gasification units: a) part of $2 \mathrm{MW}$ biomass gasification plant in Saitama, Japan [54]; b) $5 \mathrm{MW}$ pilotscale fixed bed gasification unit in Sotacarbo, Italy [55] 


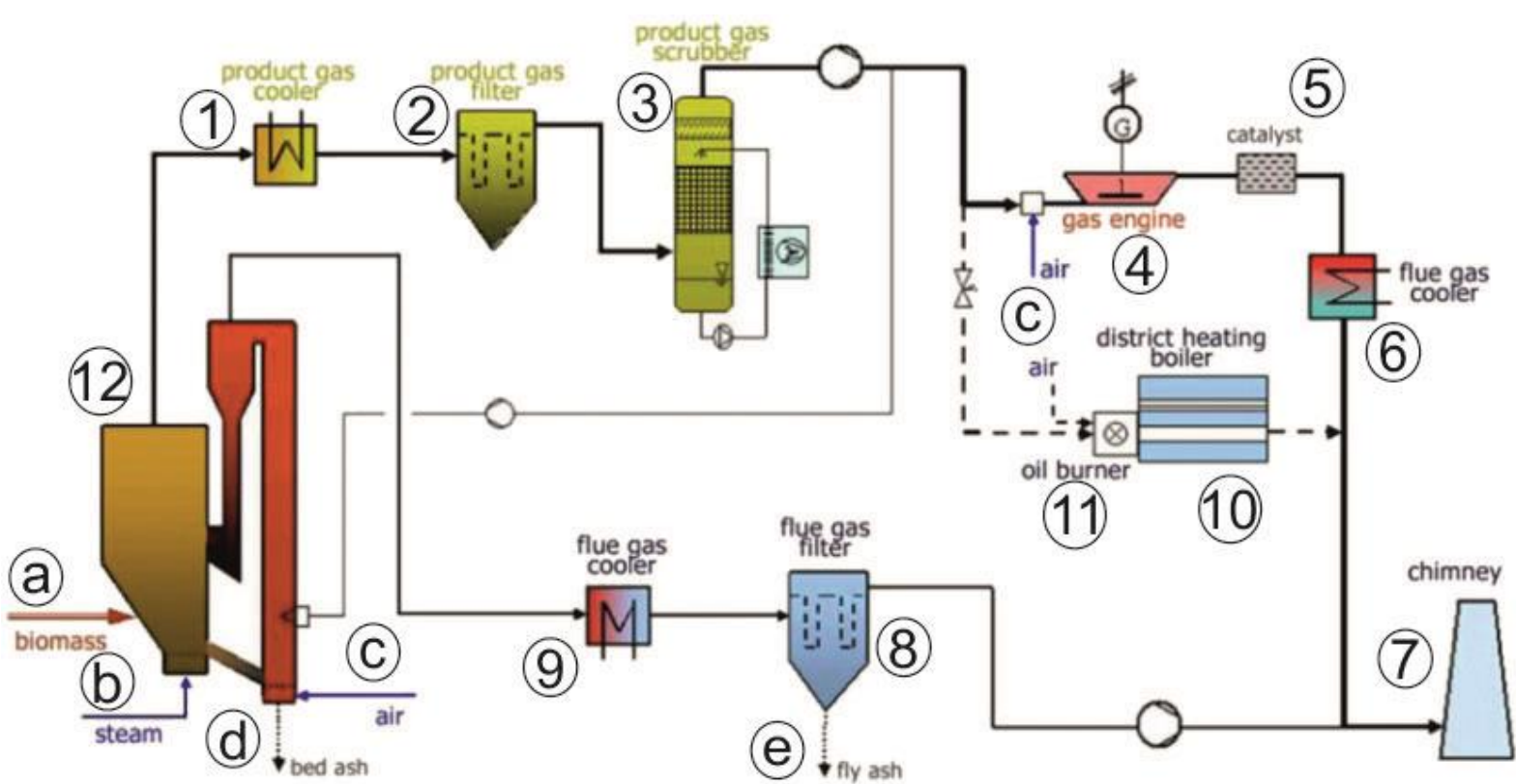

Рис. 6. Принципиальная схема газификаџионной установки DFВ в Госсинге, Австрия [57]: 1 - охладитель синтезгаза, 2 - фильтр синтез-газа, 3 - скруббер синтез-газа, 4 - газовая турбина, 5 - катализатор, 6 - охладитель дымовых газов, 7 - дымовая труба, 8 - фильтр дымовых газов, 9 - охладитель дымовых газов, 10 - котел сетевой воды, 11 - мазутная горелка, 12 - газификатор, $a$ - биомасса, $b$ - водяной пар, $c$ - воздух, $d$ - илак, $е-$ зола

Fig. 6. Principal scheme of the DFB gasification plant in Gossing, Austria [57]: 1 - product gas cooler, 2 - product gas filter, 3 - product gas scrubber, 4 - gas engine, 5 -catalyst, 6 -flue gas cooler, 7 - chimney, 8 - flue gas filter, 9 -flue gas cooler, 10 - district heating boiler, 11 - oil burner, 12 - gasificator, a - biomass, $b$ - steam, $c$-air, $d$-bed ash, $e-f l y$ ash

Отечественные технологии газификации пиролизной жидкости находятся на стадии опытноэкспериментальных разработок и исследований $[25,58]$. В указанных работах приводятся результаты расчета технологических системы, включающей в себя процессы газификации древесной биомассы, а также результаты экспериментальных исследований. Несмотря на существенно отличающуюся химию процесса газификации, её техническая реализация практически идентична процессам горения соответствующих топлив. Главным отличием является более высокое давление распыления и, как результат, необходимость установки топливных насосов.

\section{Технологии химического применения} пиролизной жидкости

Ввиду многокомпонентного состава пиролизной жидкости и наличия ряда ценных для химической промышленности веществ соответствующие технологии представляют повышенный интерес. Относительно высокая добавочная стоимость получаемых продуктов позволит повысить конкурентоспособность технологий утилизации древесной биомассы в целом. В составе пиролизной жидкости с высокой концентрацией содержатся фенолы и спирты, что обуславливает преобладание технологий получения соответствующих компонентов. Однако одной из основных проблем является достижение высокой чистоты получаемых материалов с адекватными затратами. Учитывая высокую растворимость фенолов в указанных веществах и воде, вопросы чистоты и безопасности получаемого продукта становятся особенно актуальными. Условно технологии химического производства можно разделить на две группы: требующие и не требующие высокой чистоты получаемого продукта.

\section{Технологии низкой чистоты получаемого продукта}

Одной из наиболее проработанных технологий промышленного использования пиролизной жидкости является её использование для производства вяжущего компонента для дорожного строительства. Несмотря на относительную непритязательность данного применения к составу жидкости, для эффективного использования, тем не менее, необходима простая, но многостадийная процедура подготовки жидкости (рис. $7[17,59,60])$, которая приводит к формированию загрязненных отходов (в первую очередь, воды с примесями фенолов), которые необходимо утилизировать. Возможность замещения классического связующего на производные жидкого продукта пиролиза ограничена относительно низкими значениями в 5-10 \% [17]. Вкупе с относительно невысокой добавочной стоимостью получаемого продукта наиболее распространенные технологии основаны на предположении о географической близости объекта получения пиролизной жидкости и предприятия дорожного строительства, т. к. транспортировка на большие расстояния видится нерентабельной.

Несмотря на относительную простоту (основным технологическим процессом подготовки продукта является сепарация), опыт промышленного внедрения подобных установок крайне ограничен. В зарубежной 
литературе опыт промышленного внедрения подобных технологий не описан [62, 63]. В литературном обзоре 2020 г. [63] сообщается о нахождении соответствующей технологии на стадии пилотных испытаний. В работе [61] указывается на внедрение подобных решений в ОАО «Асфальтобетонный завод», г. Красноярск в 2006 г., однако более детальной информации о практике подобного внедрения не найдено. При этом были проведены комплексные исследования данных технологических решений требованиям соответствующих ГОСТов [17], указывающие на высокую степень готовности таких технологий к практической реализации.

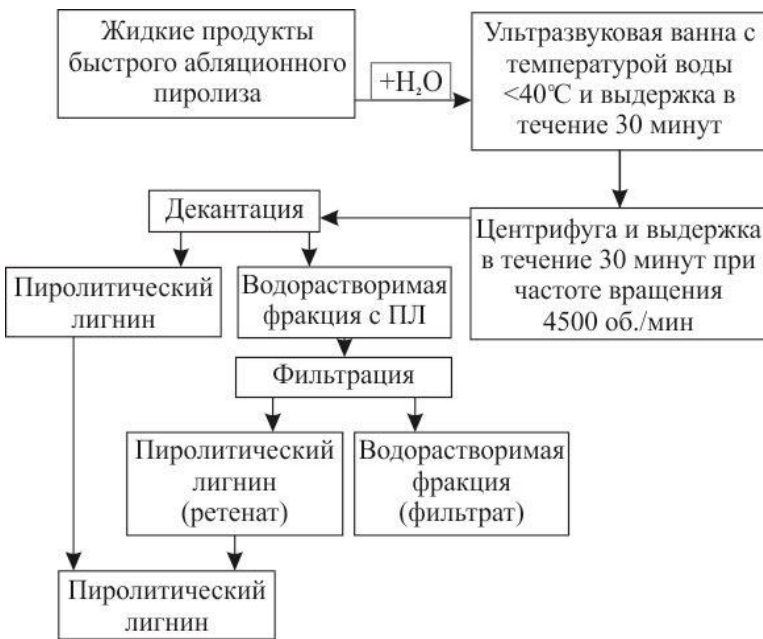

Рис. 7. Схема подготовки пиролизной жидкости для использования в качестве компонента дорожного строительства [61]

Fig. 7. Scheme of the pyrolysis liquid processing for using as a component for road building [61]

Другим подобным решением является использование пиролизной жидкости в качестве компонента древесного консерванта. Ввиду высокого содержания фенольной фракции, а также кислородсодержащих групп, использование пиролизной жидкости может привести к повышению сопротивляемости обработанной древесины к грибковому воздействию [16, 64 68] и воздействию насекомых [68]. При этом в различных работах используются разные подходы к реализации данного процесса, которые условно можно разделить на три группы:

- использование чистой сепарированной пиролизной жидкости;

- использование пиролизной жидкости в смеси с классическими компонентами (например, спиртами);

- использование сконденсированной при определенных температурах фракции пиролизной жидкости.

При реализации первого подхода [66] исследовались фунгицидные свойства пиролизной жидкости в исходном состоянии. Было установлено, что обработка древесины приводит к повышению её сопротивляемости от внешнего воздействия, однако не снижает гидрофобности древесины, что является нежелательным свойством подобных составов.
В качестве характерного примера второго подхода может служить работа [68]. В ней исследовались консервирующие свойства смесей до 20 мас. \% пиролизной жидкости с этанолом. Были получены положительные результаты в части сопротивления грибкам и насекомым (термитам), гидрофобность возросла, а старение древесины замедлилось. При этом в [67] установлено, что удовлетворительные консервирующие свойства пиролизной жидкости получены как при использовании её в смеси с 75 мас. \% этилового спирта, так и при использовании в чистом виде.

В [69] были исследованы антиокислительные свойства трех фракций пиролизной жидкости: исходной, дистилятов в температурных диапазонах 180$220{ }^{\circ} \mathrm{C}$ и $220-270{ }^{\circ} \mathrm{C}$. Установлено, что полученные при высоких температурах фракции имеют более высокую антиокислительную активность. Все указанные работы находятся на стадии лабораторных исследований и разработок.

В отечественной литературе данные работы ограничиваются принципиальным описанием технологического процесса [16]. Единственным близким решением технического характера является патент [70], описывающий получение древесной пропитки, состоящей из дистиллятов фракции термокаталитического происхождения, кипящей при $220-480{ }^{\circ} \mathrm{C}$, со смесью отработанных фракций нефтепродуктов, которые схожи по свойствам с пиролизным маслом.

Омыленная древесная смола, используемая для производства бетона, является классическим продуктом переработки пиролизной жидкости [16]. Для её применения необходимо выделение нерастворимой в воде фракции, которая затем омыливается щелочью и направляется на предприятия производства бетона.

Перспективным направлением является получение фенолформальдегидных смол для использования в древообрабатывающей промышленности в качестве связующего [71]. Данное направление представляет большой интерес ввиду относительно высокой стоимости полученного продукта и возможности снижения его токсичности. Использование токсичного фенола пиролизной жидкости для указанных целей выглядит целенаправленным и эффективным решением. Как в отечественной [72-74], так и в зарубежной литературе [75-81] приведено большое число работ в данной области.

Принцип синтеза подобных систем достаточно прост: так как фенол является водорастворимым веществом, пиролизная жидкость разбавляется водой. Далее полученная обогащенная фенолом вода отделяется от пиролизной жидкости и с помощью дистилляции путем выпаривания очищается от фенола (температура кипения фенола при атмосферном давлении составляет порядка $180{ }^{\circ} \mathrm{C}$ ). Следует отметить, что фенолы различного состава могут иметь различные температуры кипения, т. е. полученная в ходе выпаривания вода будет также загрязненной. При этом отделенная нерастворимая в воде фракция, содержащая т. н. тяжелые углеводороды, должна будет утилизироваться отдельно. 
Подход к применению пиролизной жидкости в производстве фенолформальдегидных смол схож с её применением для производства консервантов: используется либо полное, либо частичное замещение фенольной фракции производными пиролизной жидкости. При этом для её получения требуется использование формальдегида, являющегося токсичным и опасным в обращении веществом.

Так, в [81] исследовались возможности замещения чистого фенола неочищенной пиролизной жидкостью (с содержанием влаги 12 мас. \%). Установлено, что замещение более 20 мас. \% чистого фенола приводит к формированию избыточных промежуточных связей в смоле, ухудшая её адгезионные свойства. При этом отмечается, что наличие частичек биококса положительно сказывается на свойствах смолы, т. к. они служат концентраторами накопления фенольных групп. Аналогичные результаты были получены в $[75,79]$ применительно к использованию данных смол для производства древесных плит - установлено, что замещение более 25 мас. \% фенола пиролизной жидкостью приводит к значительному снижению прочностных свойств получаемого продукта. В работах $[82,83]$ указывается, что целесообразно замещение до $50 \%$ фенола. В [84] использование клея на основе фенола пиролизной жидкости (до $60 \%$ от содержания фенола в составе) позволило достичь высоких показателей прочности клеевого шва, сопоставимых с классическими составами. При этом, несмотря на то, что ряд приведенных работ использует пиролизную жидкость, полученную на промышленных предприятиях [81], данные о промышленном внедрении указанных технологических решений отсутствуют.

Технологии высокой чистоты получаемого продукта

Технологические решения по глубокой переработке пиролизной жидкости для производства продуктов с высокой добавочной стоимостью, требующих высокой степени очистки, достаточно немногочисленны. Это связано как с необходимостью реализации сложной и затратной процедуры очистки и постобработки, включающей большое число стадий (пример подобной схемы приведен на рис. 8 [85]), так и с необходимостью более строгого контроля состава используемой жидкости.

Обычно подобные решения находят достаточно ограниченное применение в виде относительно уникальных технологических решений, таких как Biolime [86-88], производство углеволокна [89] или технологий получения пищевой добавки «жидкий дым» [23, 90]. Также возможными продуктами переработки пиролизной жидкости являются очищенные до промышленного качества компоненты пиролизной жидкости [91, 92] - спирты, фенолы и др. Все приведенные данные свидетельствуют о чрезвычайной сложности такого рода технологических решений, что приводит к их более высокой стоимости относительно непосредственно установки пиролиза.
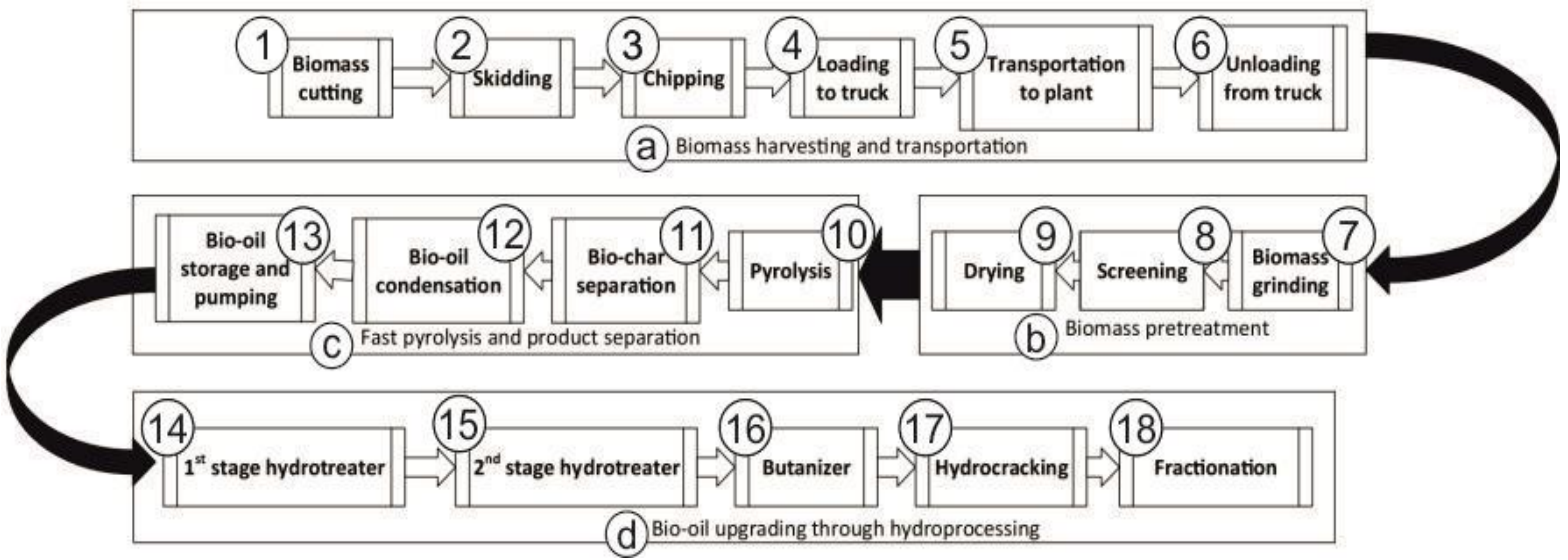

Рис. 8. Принципиальная схема производства коммерческого моторного топлива с применением пиролизной жидкости [85]: 1 - сбор биомассы, 2 - трелевка, 3 - измельчение, 4 - погрузка в грузовик, 5 - транспортировка на завод, 6 - выгрузка из грузовика, 7 - измельчение биомассы, 8 - просеивание, 9 - сушка, 10 - пиролиз, 11 - разделение биоуглерода, 12 - конденсация пиролизной жидкости, 13 - хранение и перекачка пиролизной жидкости, 14 - установка гидроочистки 1-ой ступени, 15 - установка гидроочистки 2-ой ступени, 16 - бутанизатор, 17 - гидрокрекинг, 18 - фракционирование, а - сбор и транспортировка биомассы, $b$ - предварительная обработка биомассы, с - быстрый пиролиз и разделение продуктов, $d$ - повышение качества биомассы путем гидрообработки

Fig. 8. Principal scheme of commercial engine oil production using pyrolysis liquid [85]

\section{Заключение}

Основные результаты, краткое описание и уровень готовности технологии приведены в таблице. Уровень готовности технологии оценивался согласно критериям ГОСТ Р 56861-2016 [93].

\section{Выводы}

1. В результате анализа современных публикаций отобраны и классифицированы основные технологические решения утилизации пиролизной жидкости, полученной в ходе пиролиза древесины. 
Рассмотрены технологии энергетического использования пиролизной жидкости и её переработки в химические реагенты и конечные продукты с высокой добавочной стоимостью.

Таблица. Краткое описание и технический уровень технологических решений утилизации пиролизной жидкости

Table. Short description and technological level of solutions for pyrolysis liquid utilization

\begin{tabular}{|c|c|c|}
\hline $\begin{array}{l}\text { Название } \\
\text { Name }\end{array}$ & $\begin{array}{l}\text { Краткое описание } \\
\text { Short description }\end{array}$ & $\begin{array}{l}\text { Уровень готовности } \\
\text { технологии } \\
\text { Technology readiness } \\
\text { level } \\
\end{array}$ \\
\hline $\begin{array}{l}\text { Сжигание в котлоагрегатах } \\
\text { Burning in boilers }\end{array}$ & $\begin{array}{l}\text { Прямое сжигание пиролизной жидкости в котлоагрегатах } \\
\text { Simple burning of pyrolysis liquid as fuel in boilers }\end{array}$ & $\begin{array}{l}\text { УTГ 8-9 } \\
\text { TRL 8-9 }\end{array}$ \\
\hline $\begin{array}{l}\text { Сжигание в камерах сгорания ГТУ } \\
\text { Burning in combustion chamber of gas } \\
\text { turbine units }\end{array}$ & $\begin{array}{l}\text { Сжигание в камерах сгорания ГТУ при высоких давлениях для по- } \\
\text { дачи на газовую турбину } \\
\text { Burning in combustion chamber of gas turbine units at high pressure } \\
\text { before gas turbine }\end{array}$ & \multirow{2}{*}{$\begin{array}{l}\text { УTГ } 6-7 \\
\text { TRL } 6-7\end{array}$} \\
\hline $\begin{array}{l}\text { Сжигание в поршневых двигателях } \\
\text { Combustion in reciprocating engines }\end{array}$ & $\begin{array}{l}\text { Сжигание в поршневых двигателях (дизель-генераторах) для выра- } \\
\text { ботки теплоты и электроэнергии } \\
\text { Combustion in reciprocating engines (diesel-generators) for producing } \\
\text { heat and power }\end{array}$ & \\
\hline $\begin{array}{l}\text { Газификация } \\
\text { Gasification }\end{array}$ & $\begin{array}{l}\text { Газификация для получения горючего газа } \\
\text { Gasification for obtaining combustible gas }\end{array}$ & $\begin{array}{l}\text { YTГ } 3 \\
\text { TRL } 3 \\
\end{array}$ \\
\hline $\begin{array}{l}\text { Получение связующего для дорож- } \\
\text { ного строительства } \\
\text { Producing binders for road building }\end{array}$ & $\begin{array}{l}\text { Выделение вяжущей фракции для замены битума и модификации } \\
\text { асфальта при дорожном строительстве } \\
\text { Extraction of binding fraction for substituting bitumen and modification } \\
\text { of asphalt during road building }\end{array}$ & $\begin{array}{l}\text { УTГ } 4 \\
\text { TRL } 4\end{array}$ \\
\hline $\begin{array}{l}\text { Получение древесного консерванта } \\
\text { Obtaining wood preservative }\end{array}$ & $\begin{array}{l}\text { Получения лака-консерванта для предотвращения порчи древесины } \\
\text { Obtaining preservative lacquer to prevent preliminary spoiling of wood }\end{array}$ & \multirow[b]{2}{*}{$\begin{array}{l}\text { УTГ } 3-4 \\
\text { TRL } 3-4\end{array}$} \\
\hline $\begin{array}{l}\text { Получения фенолформальдегидных } \\
\text { смол } \\
\text { Producing phenolic-formaldehyde resin }\end{array}$ & $\begin{array}{l}\text { Получения фенолформальдегидных смол путем замены промыш- } \\
\text { ленного фенола на фенол из пиролизной жидкости } \\
\text { Obtaining phenolic-formaldehyde resins by substituting industrial } \\
\text { phenol by phenol from pyrolysis liquid }\end{array}$ & \\
\hline $\begin{array}{l}\text { Получение древесной омыленной } \\
\text { смолы } \\
\text { Obtaining saponified wood resin }\end{array}$ & $\begin{array}{l}\text { Нерастворимая в воде фракция пиролизной жидкости омыливается } \\
\text { щелочью } \\
\text { Insoluble in water fraction of pyrolysis liquid is saponified by alkali }\end{array}$ & $\begin{array}{l}\text { УTГ } 3 \\
\text { TRL } 3\end{array}$ \\
\hline $\begin{array}{l}\text { Технологии комплексной переработки } \\
\text { Complex processing technologies }\end{array}$ & $\begin{array}{l}\text { Дистилляция пиролизной жидкости для выделения отдельных ком- } \\
\text { понент для химической промышленности и потребителя } \\
\text { Pyrolysis liquid distillation to extract components for chemical industry } \\
\text { and consumers }\end{array}$ & $\begin{array}{l}\text { УТГ 2-3 } \\
\left(\text { УTГ } 7-8^{*}\right) \\
\text { TRL 2-3 } \\
\left(\text { TRL } 7-8^{*}\right) \\
\end{array}$ \\
\hline
\end{tabular}

*Для отдельных технологических решений, приведенных в [36, 90].

*For some technological solutions presented in [36, 90].

2. Технологии энергетического использования пиролизной жидкости имеют достаточно высокую степень проработки и подготовленности для технического внедрения, выраженную в наличии опытно-промышленных образцов и даже коммерческих решений. Основные отличия пиролизной жидкости от классического жидкого топлива заключаются в более высокой вязкости, кислотности, влажности, волатильности состава и низкой стабильности теплоты сгорания. Одним из наиболее существенных недостатков является высокая кислотность, требующая модификации топливного тракта с использованием коррозионностойких материалов. Ввиду незначительности отличий соответствующих технологий от классических решений в настоящее время имеются комплексные коммерческие решения для сжигания пиролизной жидкости в котельных агрегатах, камерах сгорания и поршневых двигателях.

3. К общим недостаткам технологий разделения пиролизной жидкости на индивидуальные вещества для химических производств или конечного по- требления относят сложность соответствующих технологических схем и зачастую необходимость вовлечения дополнительных реагентов в процесс. Лучше всего проработаны наиболее простые технологические решения, основанные на переработке пиролизной жидкости с получением связующего для дорожного строительства, консерванта для древесины или фенолформальдегидных смол. Однако практическая реализация подобных технологий достаточно ограничена и в большинстве случаев пока не выходит за рамки лабораторных и экспериментальных исследований. Более сложные технологические решения нашли крайне ограниченное применение отдельными производителями конкретного продукта.

Работа была реализована при финансовой поддержке по дополнительному контракту № 075-03-2021-138/3 о субсидии из федерального бюджета на финансовую поддержку реализации государственного задания по оказанию государственных услуг (внутренний номер 075-GZ/X4141/687/3). 


\section{СПИСОК ЛИТЕРАТУРЫ}

1. Improving environmental and energy efficiency in wood transportation for a carbon-neutral forest industry / T. Palander, H. Haavikko, E. Kortelainen, K. Kärhä, S.A. Borz // Forests. 2020. - V. 11. - № 11. - P. 1-17.

2. Thermochemical processing of woody biomass: a review focused on energy-driven applications and catalytic upgrading / J.C. Solarte-Toro, J.A. González-Aguirre, J.A. Poveda Giraldo, C.A. Cardona Alzate // Renewable and Sustainable Energy Reviews. - 2021. - V. 136. - P. 110376.1-16.

3. Barmore T. Biomass pyrolysis: past, present, and future // Environment, Development and Sustainability. - 2020. - V. 22. № 1. - P. 17-32.

4. Pre-treatment of furniture waste for smokeless charcoal production / P. Kazimierski, P. Hercel, K. Januszewicz, D. Kardaś // Materials. 2020. - V. 13. - № 14. - P. 1-11.

5. Investigation of the influence of ultrasonic treatment on the sorption properties of the pyrolysis product of wood waste I.A. Nasyrov, G.V. Mavrin, D.D. Fazullin, I.G. Shaikhiev // Journal of Physics: Conference Series. - 2019. - V. 1347. - № 1. P. 012080.1-5

6. Cost-effective sulfurized sorbents derived from one-step pyrolysi of wood and scrap tire for elemental mercury removal from flue gas / Y. Xu, G. Luo, Q. Zhang, Z. Li, S. Zhang, W. Cui // Fuel. 2021. - V. 285. - P. 119221.1-8.

7. Evaluation of biochar post-process treatments to produce soil enhancers and phosphorus fertilizers at a single plant / M. Laghari, D.S. Müller-Stöver, M. Puig-Arnavat, T.P. Thomsen, U.B. Henriksen // Waste and Biomass Valorization. - 2021. V. 12. - № 10. - P. 5517-5532.

8. Fuel sensitivity of biomass cookstove performance / L. Deng, D. Torres-Rojas, M. Burford, T.H. Whitlow, J. Lehmann, E.M. Fisher // Applied Energy. - 2018. - V. 215. - P. 13-20.

9. Life cycle assessment of charcoal production and electricity generation from eucalyptus in an industrial batch kiln M.M. Vicente Leme, O.J. Venturini, E.E. Silva Lora, W. de Almeida M.H. Rocha, T. Andrade da Cunha Dias, O.A. del Olmo // Renewable Energy. - 2021. - V. 180. - P. 232-244.

10. Richard T.L. Challenges in scaling up biofuels infrastructure // Science. - 2010. - V. 329. - № 5993. - P. 793-796.

11. Norms, standards, and legislation for fast pyrolysis bio-oils from lignocellulosic biomass / A. Oasmaa, B. van de Beld, P. Saari, D.C. Elliott, Y. Solantausta // Energy \& Fuels. - 2015. - V. 29. № 4. - P. 2471-2484.

12. Efika C.E., Onwudili J.A., Williams P.T. Influence of heating rates on the products of high-temperature pyrolysis of waste wood pellets and biomass model compounds // Waste Management. 2018. - V. 76. - P. 497-506.

13. Evaluation of the physiochemical development of biochars obtained from pyrolysis of wheat straw, timothy grass and pinewood: Effects of heating rate / P. Mohanty, S. Nanda, K.K. Pant, S. Naik, J.A. Kozinski, A.K. Dalai // Journal of Analytical and Applied Pyrolysis. - 2013. - V. 104. - P. 485-493.

14. Characteristic studies on the pyrolysis products from hydrolyzed Canadian lignocellulosic feedstocks / S. Nanda, R. Azargohar, J.A. Kozinski, A.K. Dalai // Bioenergy Research. - 2014. - V. 7. № 1. - P. 174-191.

15. Understanding the pyrolysis behavior of agriculture, forest and aquatic biomass: Products distribution and characterization G. Wang, B. Fan, H. Chen, Y. Li // Journal of the Energy Institute. 2020. - V. 93. - № 5. - P. 1892-1900.

16. Башкиров В.Н., Бикбулатова Г.М., Гатауллина А.А. Перспективы промышленного использования жидких продуктов быстрого пиролиза растительной биомассы // Евразийское Научное Объединение. - 2018. - Т. 46. - № 12-3. - С. 207-210.

17. Файзрахманова Г.М. и др. Использование древесной пиролизной жидкости для получения химических продуктов // Вестник Казанского технологического университета. - 2013. T. 16. - № 8. - C. 312-314.

18. Standard liquid fuel for industrial boilers from used wood / C. Lindfors, A. Oasmaa, A. Välimäki, T. Ohra-aho, H. Punkkinen, C. Bajamundi, K. Onarheim // Biomass and Bioenergy. - 2019. V. 127. - P. 105265.1-8.
19. Albert-Green S., Thomson M.J. The effects of nozzle design on the combustion of wood-derived fast pyrolysis oil // Biomass and Bioenergy. - 2018. - V. 117. - P. 102-114.

20. Krumdieck S.P., Daily J.W. Evaluating the feasibility of biomass pyrolysis oil for spray combustion applications // Combustion Science and Technology. - 1998. - V. 134. - № 1-6. - P. 351-365.

21. Co-firing of fast pyrolysis bio-oil and heavy fuel oil in a $300-\mathrm{kWth}$ furnace / S.S. Hou, W.C. Huang, F.M. Rizal, T.H. Lin // Applied Sciences (Switzerland). - 2016. - V. 6. - № 11. - P. 326.1-11.

22. Энергетическое использование пиролизной жидкости / С.А. Забелкин, А.Н. Грачёв, В.Н. Башкиров, Ф.И. Мулламухаметов // Вестник Казанского технологического университета. -2010 . - № 10. - С. 369-374.

23. Loganathan K., Manoharan C. Evaluation of performance and emission features of jatropha biodiesel-turpentine blend as green fuel // Thermal Science. - 2017. - V. 21. - № 1. - P. 615-625.

24. Nyashina G.S., Vershinina K.Y., Strizhak P.A. Impact of microexplosive atomization of fuel droplets on relative performance indicators of their combustion // Fuel Processing Technology. 2020. - V. 201. - № October 2019. - P. 106334.1-14.

25. Технологическая схема газификации жидкого продукта контактного пиролиза / Д.В. Тунцев, Р.Г. Сафин, А.М. Касимов, Р.Г. Хисматов, З.Г. Саттарова // Вестник Казанского технологического университета. - 2013. - Т. 16. - № 19. - С. 139-141.

26. Fortum 2021. URL: https://www.fortum.ru/ (дата обращения 11.11.2021).

27. Emissions from a fast-pyrolysis bio-oil fired boiler: comparison of health-related characteristics of emissions from bio-oil, fossil oil and wood / O. Sippula, K. Huttunen, J. Hokkinen, S. Kärki, H. Suhonen, T. Kajolinna, M. Kortelainen, T. Karhunen, P. Jalava, O. Uski, P. Yli-Pirilä, M.R. Hirvonen, J. Jokiniemi // Environmental Pollution. - 2019. - V. 248. - P. 888-897.

28. Ensyn Inc. 2021. URL: http://www.ensyn.org/en/home/ (дата обращения 11.11.2021).

29. Valmet 2021. URL: https://www.valmet.com/ru/ (дата обращения 11.11.2021)

30. Stork Inc. 2021. URL: https://www.stork.com/en (дата обращения 11.11.2021).

31. Vershinina K.Y., Shlegel N.E., Strizhak P.A. Impact of environmentally attractive additives on the ignition delay times of slurry fuels: experimental study // Fuel. - 2019. - V. 238. P. 275-288.

32. Энергетическое использование жидких продуктов быстрого пиролиза древесины / С.А. Забелкин, Д.В. Тунцев, А.Н. Грачев, В.Н. Башкиров // Биоэнергетика и биотехнологии. 2010. - T. 4. - C. 79-84.

33. Lupandin V., Thamburaj R., Nikolayev A. Test results of the OGT2500 gas turbine engine running on alternative fuels: BioOil, Ethanol, Biodiesel and Crude Oil // ASME Turbo Expo 2005: Power for Land, Sea, and Air. - Nevada: International Gas Turbine Institute, 2005. - P. 421-426

34. Opra Turbines Inc. 2021. URL: https://www.opraturbines.com/ru/ (дата обращения 11.11.2021).

35. Improved CFD predictions of pyrolysis oil combustion using advanced spray measurements and numerical models / E. van Beurden, A. Pozarlik, B. Putra, G. Brem, T. Bouten, J. Withag, L.U. Axelsson // ASME Turbo Expo 2021: Turbomachinery Technical Conference and Exposition, GT 2021. - Virtual: American Society of Mechanical Engineers, 2021. - V. 6. - P. 1-12.

36. Experimental and numerical investigation of the application of fast pyrolysis oil in a gas turbine combustor / T. Bouten, J. Withag, L. Axelsson, B. Putra, A. Pozarlik, G. Brem, C. Benesch, T. Brunner, I. Obernberger // $28^{\text {th }}$ European Biomass Conference and Exhibition, e-EUBCE 2020. - Virtual: ETA-Florence Renewable Energies, 2020. - P. 957-965.

37. Seljak T., Širok B., Katrašnik T. Advanced fuels for gas turbines: fuel system corrosion, hot path deposit formation and emissions // Energy Conversion and Management. - 2016. - V. 125. - P. 40-50.

38. Chiaramonti D., Oasmaa A., Solantausta Y. Power generation using fast pyrolysis liquids from biomass // Renewable and Sustainable Energy Reviews. - 2007. - V. 11. - № 6. - P. 1056-1086.

39. VTT Technical Research Centre of Finland Ltd. 2021. URL: https://www.vttresearch.com/en (дата обращения 11.11.2021).

40. Wärtsilä Corp. 2021. URL: https://www.wartsila.com/ (дата обращения 11.11.2021) 
41. Solantausta Y., Nylund N.O., Gust S. Use of pyrolysis oil in a test diesel engine to study the feasibility of a diesel power plant concept // Biomass and Bioenergy. - 1994. - V. 7. - № 1-6. P. 297-306.

42. Yang S.I., Wu M.S. The droplet combustion and thermal characteristics of pinewood bio-oil from slow pyrolysis // Energy. - 2017. - V. 141. - P. 2377-2386.

43. Kim T.Y., Lee S., Kang K. Performance and emission characteristics of a high-compression-ratio diesel engine fueled with wood pyrolysis oil-butanol blended fuels // Energy. - 2015. V. 93. - P. 2241-2250.

44. Performance and emission charactertistics of an IDI diese generator fueled with wood pyrolysis oil-butanol blended fuels / S. Lee, T.Y. Kim, K. Kang, I. Park, M. Kim, J. Lim // Transactions of The Korean Society of Automotive Engineers - 2017 - V. 25. № 3. - P. 380-388,

45. Lee S., Kim Y., Kang K. The role of exergy in energy and the environment. - Cham: Springer Verlag, 2018. - $906 \mathrm{p}$.

46. Lee S., Kim T.Y. Feasibility study of using wood pyrolysis oilethanol blended fuel with diesel pilot injection in a diesel engine // Fuel. - 2015. - V. 162. - P. 65-73.

47. Лиханов В.А., Козлов А.Н., Арасланов М.И. Влияние применения этанола и рапсового масла на показатели процесса сгорания и сажесодержания в цилиндре тракторного дизеля $2 Ч$ 10,5/12,0 в зависимости от изменения нагрузки // Вестник чувашской государственной сельскохозяйственной академии. 2018. - T. 4. - № 1. - C. 59-64.

48. BTG Biomass Technology Group 2021. URL https://www.btgworld.com/ (дата обращения 11.11.2021).

49. К вопросу расширения использования газа в качестве моторного топлива / С.В. Дунаев, А.В. Исаев, А.В. Лесин, В.П. Попов, К.И. Федин // Мир нефтепродуктов. Вестник нефтяных компаний. - 2017. - № 1. - С. 10-14.

50. Чирков В.Г. Мини-ТЭС на пиролизном топливе // Теплоэнергетика. - 2007. - № 8. - С. 35-39.

51. Результаты экспериментального исследования процесса газификации жидкого продукта быстрого пиролиза древесных отходов лесной промышленности / Д.В. Тунцев, Р.Г. Хисматов, А.М. Касимов, И.С. Романчева, А.С. Савельев // Актуальные направления научных исследований XXI века: теория и практика. - Воронеж: Воронежский государственный лесотехнический университет им. Г.Ф. Морозова, 2016. - С. 352-356.

52. Pyrolysis-catalytic upgrading of bio-oil and pyrolysis-catalytic steam reforming of biogas: a review / M. Abou Rjeily, C. Gennequin, H. Pron, E. Abi-Aad, J.H. Randrianalisoa // Environmental Chemistry Letters. - 2021. - V. 19. - № 4. P. $2825-2872$.

53. Towards practical application of gasification: a critical review from syngas and biochar perspectives / S. You, Y.S. Ok, D.C.W. Tsang, E.E. Kwon, C.H. Wang // Critical Reviews in Environmental Science and Technology. - 2018. - V. 48. № 22-24. - P. 1165-1213.

54. Baba Y., Pandyaswargo A.H., Onoda H. An analysis of the current status of woody biomass gasification power generation in Japan // Energies. - 2020. - V. 13. - № 18. - P. 4903.1-13.

55. Syngas production, clean-up and wastewater management in a demo-scale fixed-bed updraft biomass gasification unit / G. Calì, P. Deiana, C. Bassano, S. Meloni, E. Maggio, M. Mascia, A. Pettinau // Energies. - 2020 - V. 13. - № 10. - P. 2594.1-15.

56. Siemens 2004-2021. URL: https://new.siemens.com/ru/ru.html (дата обращения 11.11.2021).

57. Biomass gasification cogeneration - a review of state of the art technology and near future perspectives / J. Ahrenfeldt, T.P. Thomsen, U. Henriksen, L.R. Clausen // Applied Thermal Engineering. - 2013. - V. 50. - № 2. - P. 1407-1417.

58. Касимов А.М. Совершенствование технологии и оборудования термохимической перереботки древесных отходов в выококалорийный генераторный газ: автореф. дис. ... канд. тех. наук. - Казань, 2017. - 16 с.

59. Файзрахманова Г.М. Модификация битумных вяжущих жидкими продуктами быстрого абляционного пиролиза древесины: автореф. дис. ... канд. тех. наук. - Казань, 2015. - 16 с.

60. Разработка технологии получения органического вяжущего для дорожного строительства с использованием продуктов термической переработки биомассы дерева / Г.М. Файзрахма- нова, С.А. Забелкин, А.Н. Грачев, В.Н. Башкиров // Вестник Приамурского государственного университета им. ШоломАлейхема. - 2015. - Т. 19. - № 2. - С. 79-85.

61. Киселев В.П. Комплексное использование отходов химической переработки биомассы дерева и других вторичных ресурсов в производстве композиционных вяжущих и материалов, полученных на их основе: автореф. дис. ... д-ра техн. наук. - Красноярск, 2006. -43 с.

62. Modification of bitumen binder by the liquid products of wood fast pyrolysis / S. Zabelkin, G. Bikbulatova, A. Grachev, V. Bashkirov, S. Burenkov, A. Makarov // Road Materials and Pavement Design. - 2019. - V. 20. - № 5. - P. 1182-1200

63. Preparation process of bio-oil and bio-asphalt, their performance, and the application of bio-asphalt: A comprehensive review / H. Wang, Z. Ma, X. Chen, M.R. Mohd Hasan // Journal of Traffic and Transportation Engineering. - 2020. - V. 7. - № 2. - P. 137-151.

64. Bio-based wood preservatives: their efficiency, leaching and ecotoxicity compared to a commercial wood preservative A. Barbero-López, J. Akkanen, R. Lappalainen, S. Peräniemi, A. Haapala // Science of the Total Environment. - 2021. V. 753. - P. 142013.1-7.

65. Mazela B. Fungicidal value of wood tar from pyrolysis of treated wood // Waste Management. - 2007. - V. 27. - № 4. - P. 461-465.

66. Preliminary study on fungicide and sorption effects of fast pyrolysis liquids used as wood preservative / D. Meier, B. Andersons, I. Irbe, J. Chirkova, O. Faix // Progress in Thermochemical Biomass Conversion. - Hoboken: Wiley Blackwell, 2008. - P. 1550-1563.

67. Evaluation of the antifungal effects of bio-oil prepared with lignocellulosic biomass using fast pyrolysis technology / K.H. Kim, H.S. Jeong, J.Y. Kim, G.S. Han, I.G. Choi, J.W. Choi // Chemosphere. - 2012. - V. 89. - № 6. - P. 688-693.

68. Chemical composition and efficiency of bio-oil obtained from giant cane as a wood preservative / A. Temiz, S. Akbas, D. Panov, N. Terziev, M.H. Alma, S. Parlak, G. Kose // BioResources. 2013. - V. 8. - № 2. - P. 2084-2098.

69. Hassan E.B., El-Giar E.M., Steele P. Evaluation of the antioxidant activities of different bio-oils and their phenolic distilled fractions for wood preservation // International Biodeterioration and Biodegradation. - 2016. - V. 110. - P. 121-128.

70. Нефтяная антисептическая жидкость для пропитки древесины: пат. Рос. Федерация, № 2455154C1, заявл. 29.10.2010; опубл. 07.10.2012. Бюл. № 19. -6 c

71. Ma Y., Gong X., Wang C. Research progress in wood adhesives // Chemistry and Industry of Forest Products. - 2020. - V. 40. № 2. - P. 1-15.

72. Определение влияния степени замещения фенола жидкими продуктами пиролиза древесины на прочность фенолоформальдегидной смолы / А.Р. Валеева, А.Н. Грачев, С.А. Забелкин, В.Н. Башкиров, А.И. Сабирзянова // Деревообрабатывающая промышленность. - 2020. - № 1. - С. 88-95.

73. Модификация фенолоформальдегидных смол жидкими продуктами пиролиза древесины и изучение их клеящей способности / С.А. Забелкин, А.Н. Грачёв, В.Н. Башкиров, Е.Н. Черезова // Вестник Казанского технологического университета. 2010. - T. 8. - C. $440-444$

74. Модификация фенолоформальдегидных смол жидкими продуктами пиролиза древесины / С.А. Забелкин, А.Н. Грачёв, В.Н. Башкиров, Е.Н. Черезова // Вестник Казанского технологического университета. - 2014. - Т. 17. - № 10. - С. 97-100.

75. Mao A., Shi S.Q., Steele P. Flakeboard bonded with polymeric diphenylmethane diisocyanate/ bio-oil adhesive systems // Forest Products Journal. - 2011. - V. 61. - № 3. - P. 240-245.

76. Life cycle assessment of plywood manufacturing process in China/ L. Jia, J. Chu, L. Ma, X. Qi, A. Kumar // International Journal of Environmental Research and Public Health. - 2019. - V. 16. № 11. - P. 2037.1-10.

77. The preparation and characterization of pyrolysis bio-oilresorcinol-aldehyde resin cold-set adhesives for wood construction / X. Ren, H. Cai, H. Du, J. Chang // Polymers. - 2017. - V. 9. № 6. -P. 232.1-11.

78. Ayrilmis N., Özbay G. Propriedades tecnológicas de compensado de madeira colada com resina de resol fenol-formaldeído sintetizada com bio-óleo // Cerne. - 2017. - V. 23. - № 4. P. 493-500. 
79. Evaluation of phenol-formaldehyde resins modified and blended with pyrolysis bio-oil for plywood / A. Mao, W. Xu, E. Xi, Q. Li, H. Wan // Forest Products Journal. - 2018. - V. 68. - № 2. P. 113-119.

80. The use of environmentally friendly bio-oil in the production of phenol formaldehyde (PF) resin / N.N. Omar, I.S. Mustafa, N. Abdullah, R. Hashim // Pertanika Journal of Science and Technology. - 2018. - V. 26. - № 1. - P. 177-192.

81. Production of novolac resin after partial substitution of phenol from bio-oil / S. Adhikari, M. Auad, B. Via, A. Shah, V. Patil // Transactions of the ASABE. - 2020. - V. 63. - № 4. - P. 901-912.

82. Dong F., Wang M., Wang Z. Bio-oil as substitute of phenol for synthesis of resol-type phenolic resin as wood adhesive // International Journal of Chemical Reactor Engineering. - 2018. V. 16. - № 3. - P. 20170107.1-8.

83. Preparation and characterization of epoxy resin cross-linked with high wood pyrolysis bio-oil substitution by acetone pretreatment / Y. Liu, B.K. Via, Y. Pan, Q. Cheng, H. Guo, M.L. Auad, S. Taylor // Polymers. - 2017. - V. 9. - № 3. - P. 106.1-14.

84. Резольная феноло-формальдегидная смола с добавлением нейтрализованных жидких продуктов быстрого пиролиза древесины березы / С.А. Забелкин, А.Н. Грачев, Г.М. Бикбулатова, А.Е. Яковлева, А.А. Макаров, В.Н. Башкиров // Клеи. Герметики. Технологии. - 2017. - № 10. - С. 19-23.

85. A techno-economic assessment of renewable diesel and gasoline production from aspen hardwood / M. Patel, A.O. Oyedun, A. Kumar, R. Gupta // Waste and Biomass Valorization. - 2019. V. 10. - № 10. - P. 2745-2760.

86. Pisupati S. V., Bhalla S. Influence of calcium content of biomassbased materials on simultaneous $\mathrm{NO}_{\mathrm{x}}$ and $\mathrm{SO}_{2}$ reduction //
Environmental Science and Technology. - 2008. - V. 42. - № 7. P. 2509-2514.

87. Pisupati S. V., Bhalla S. Numerical modeling of NOx reduction using pyrolysis products from biomass-based materials // Biomass and Bioenergy. - 2008. - V. 32. - № 2. - P. 146-154.

88. Simultaneous $\mathrm{SO}_{2} / \mathrm{NO} / \mathrm{N}_{2} \mathrm{O}$ control in coal fired systems using BioLime $^{\mathrm{TM}}$ / G.A. Simons, W.J. Place, K.H. Oehr, J. Zhou, S.V. Pisupati, M.A. Woitowicz, R. Bassilakis // Proceedings of the International Technical Conference on Coal Utilization \& Fuel Systems. - Florida: Coal \& Slurry Technology Assoc., 1997. P. 739-749.

89. Vélez D.C.P., Magalhães W.L.E., Capobianco G. Carbon fiber from fast pyrolysis bio-oil // Science and Technology of Materials. 2018. - V. 30. - P. 16-22.

90. Transforming biomass pyrolysis technologies to produce liquid smoke food flavouring / X. Xin, K. Dell, I.A. Udugama, B.R. Young, S. Baroutian // Journal of Cleaner Production. 2021. - V. 294. - P. 125368.1-37.

91. Онофрейчук А.О. Фракционная перегонка пиролизного дистиллята // Булатовские чтения. - 2018. - № 5. - С. 238-240.

92. Jacobson K., Maheria K.C., Kumar Dalai A. Bio-oil valorization: a review // Renewable and Sustainable Energy Reviews. - 2013. V. 23. - P. 91-106.

93. ГОСТ Р 56861-2016. Система управления жизненным циклом. Разработка концепции изделия и технологий. Общие положения. - М.: Стандартинформ, 2019. - 10 с.

Поступила 01.12.2021 2.

\section{Информация об авторах}

Слюсарский К.B., кандидат физико-математических наук, доцент НОЦ И.Н. Бутакова Инженерной школы энергетики Национального исследовательского Томского политехнического университета.

Ларионов К.Б., кандидат технических наук, заведующий лабораторией Кузбасского государственного технического университета им. Т.Ф. Горбачева.

Ивашкина $\boldsymbol{E} . \boldsymbol{H}$., доктор технических наук, профессор отделения химической инженерии Инженерной школы природных ресурсов Национального исследовательского Томского политехнического университета.

Заворин $\boldsymbol{A . C}$., доктор технических наук, Заведующий кафедрой - руководитель научно-образовательного центра И.Н. Бутакова на правах кафедры Инженерной школы энергетики Национального исследовательского Томского политехнического университета.

Губин B.E., кандидат технических наук, доцент НОЦ И.Н. Бутакова Инженерной школы энергетики Национального исследовательского Томского политехнического университета. 
UDC 620.952

\title{
TECHNOLOGICAL SOLUTIONS FOR UTILIZATION OF LIQUID PRODUCTS OF SLOW WOOD BIOMASS PYROLYSIS
}

\author{
Konstantin V. Slyusarsky ${ }^{1}$, \\ slyuskonst@gmail.com
}

Kirill B. Larionov², laryk070@gmail.com

Elena N. Ivashkina ${ }^{1}$, ivashkinaen@tpu.ru

\author{
Alexander S. Zavorin ${ }^{1}$, \\ zavorin@tpu.ru \\ Vladimir E. Gubin', \\ gubin@tpu.ru \\ ${ }^{1}$ National Research Tomsk Polytechnic University, \\ 30, Lenin avenue, Tomsk, 634050, Russia. \\ 2 T.F. Gorbachev Kuzbass State Technical University, \\ 28, Vesennyaya street, Kemerovo, 650000, Russia.
}

The relevance of the article is caused by the growing interest in biomass pyrolysis technologies to reduce the carbon footprint of its processing. To increase the economic and energy efficiency of these technological solutions, it is necessary to maximize the full use of all material and energy flows, one of which is the formation of liquid pyrolysis products (the so-called pyrolysis liquid or bio-oil).

The main aim of the research is determination of technological solutions for the disposal of liquid pyrolysis products of woody biomass with the determination of the level of technical readiness.

Methods: analytical literature review of literature data using materials from the RSCl, Scopus and Web of Science databases; the level of technical readiness was assessed according to the TRL scale.

Results. The main methods of utilization of the pyrolysis liquid obtained as a result of slow pyrolysis of woody biomass were identified, classified and described. A review of technological solutions and research works in the field of disposal of liquid products of slow pyrolysis was made. The most common technologies for converting a pyrolysis liquid to obtain thermal and/or electrical energy were direct combustion in burners of boilers and heat generators, in the combustion chamber of gas turbine engines, gasification to produce synthesis gas, and the use in piston engines as fuel. Technologies for obtaining chemical products and substances were divided into simple (obtaining components for road construction, wood preservative, phenol-formaldehyde and saponified resins, etc.) and complex, requiring complex multistage processing. It was established that the energy application of this product has the highest level of technical readiness, while the chemical application technologies have the highest economic efficiency, despite the relatively low level of readiness. Commercial solutions for the disposal of pyrolysis fluid are available in the field of combustion in burners and diesel generators.

\section{Key words:}

Pyrolysis, biomass, incineration, combustion, bio-oil, pyrolysis fluid, bio-char.

The study was realized with financial support according to additional contract no. 075-03-2021-138/3 on subsidy from federal budget for financial support of governmental task realization for governmental services (internal number 075-GZ/X4141/687/3).

\section{REFERENCES}

1. Palander T., Haavikko H., Kortelainen E., Kärhä K., Borz S.A. Improving environmental and energy efficiency in wood transportation for a carbon-neutral forest industry. Forests, 2020, vol. 11, no. 11,1194 . pp. 1-17.

2. Solarte-Toro J.C., González-Aguirre J.A., Poveda Giraldo J.A., Cardona Alzate C.A. Thermochemical processing of woody biomass: a review focused on energy-driven applications and catalytic upgrading. Renewable and Sustainable Energy Reviews, 2021, vol. 136, pp. 110376.1-16.

3. Barmore T. Biomass pyrolysis: past, present, and future. Environment, Development and Sustainability, 2020, vol. 22, no. 1, pp. 17-32.

4. Kazimierski P., Hercel P., Januszewicz K., Kardaś D. Pretreatment of furniture waste for smokeless charcoal production. Materials, 2020, vol. 13, no. 14, 3188. pp. 1-11.

5. Nasyrov I.A., Mavrin G. V., Fazullin D.D., Shaikhiev I.G. Investigation of the influence of ultrasonic treatment on the sorption properties of the pyrolysis product of wood waste. Journal of Physics: Conference Series, 2019, vol. 1347, no. 1, pp. 012080.1-5.

6. Xu Y., Luo G., Zhang Q., Li Z., Zhang S., Cui W. Cost-effective sulfurized sorbents derived from one-step pyrolysis of wood and scrap tire for elemental mercury removal from flue gas. Fuel, 2021, vol. 285, pp. 119221.1-8.

7. Laghari M., Müller-Stöver D.S., Puig-Arnavat M., Thomsen T.P., Henriksen U.B. Evaluation of biochar post-process treatments to produce soil enhancers and phosphorus fertilizers at a single plant. Waste and Biomass Valorization, 2021, vol. 12, no. 10, pp. 5517-5532.

8. Deng L., Torres-Rojas D., Burford M., Whitlow T.H., Lehmann J., Fisher E.M. Fuel sensitivity of biomass cookstove performance. Applied Energy, 2018, vol. 215, pp. 13-20.

9. Vicente Leme M.M., Venturini O.J., Silva Lora E.E., de Almeida W., Rocha M.H., Andrade da Cunha Dias T., del Olmo O.A. Life cycle assessment of charcoal production and electricity generation from eucalyptus in an industrial batch kiln. Renewable Energy, 2021, vol. 180, pp. 232-244. 
10. Richard T.L. Challenges in scaling up biofuels infrastructure. Science, 2010, vol. 329, no. 5993, pp. 793-796.

11. Oasmaa A., van de Beld B., Saari P., Elliott D.C., Solantausta Y. Norms, standards, and legislation for fast pyrolysis bio-oils from lignocellulosic biomass. Energy \& Fuels, 2015, vol. 29, no. 4, pp. $2471-2484$

12. Efika C.E., Onwudili J.A., Williams P.T. Influence of heating rates on the products of high-temperature pyrolysis of waste wood pellets and biomass model compounds. Waste Management, 2018 vol. 76, pp. 497-506.

13. Mohanty P., Nanda S., Pant K.K., Naik S., Kozinski J.A., Dalai A.K. Evaluation of the physiochemical development of biochars obtained from pyrolysis of wheat straw, timothy grass and pinewood: Effects of heating rate. Journal of Analytical and Applied Pyrolysis, 2013, vol. 104, pp. 485-493.

14. Nanda S., Azargohar R., Kozinski J.A., Dalai A.K. Characteristic studies on the pyrolysis products from hydrolyzed canadian lignocellulosic feedstocks. Bioenergy Research, 2014, vol. 7, no. 1, pp. 174-191.

15. Wang G., Fan B., Chen H., Li Y. Understanding the pyrolysis behavior of agriculture, forest and aquatic biomass: Products distribution and characterization. Journal of the Energy Institute, 2020, vol. 93, no. 5, pp. 1892-1900.

16. Bashkirov V.N., Bikbulatova G.M., Gataullina A.A. Perspektivy promyshlennogo ispolzovaniya zhidkikh productov bystrogo piroliza rastitelnoy biomassy [Prospects for industrial use of liquid products of rapid pyrolysis of plant biomass]. Evraziyskoe nauchnoe obyedinenie, 2018, vol. 46, no. 12-3, pp. 207-210.

17. Fayzrakhmanova G.M. Zabelkin S.A., Grachev A.N., Bashkirov V.N., Makarov A.A. Use of wood pyrolysis liquid for obtaining a binder component for road construction. Bulletin of the Technological University, 2013, vol. 16, no. 8, pp. 312-314. In Rus.

18. Lindfors C., Oasmaa A., Välimäki A., Ohra-aho T., Punkkinen H., Bajamundi C., Onarheim K. Standard liquid fuel for industrial boilers from used wood. Biomass and Bioenergy, 2019, vol. 127, pp. 105265.1-8.

19. Albert-Green S., Thomson M.J. The effects of nozzle design on the combustion of wood-derived fast pyrolysis oil. Biomass and Bioenergy, 2018, vol. 117, pp. 102-114.

20. Krumdieck S.P., Daily J.W. Evaluating the feasibility of biomass pyrolysis oil for spray combustion applications. Combustion Science and Technology, 1998, vol. 134, no. 1-6, pp. 351-365.

21. Hou S.S., Huang W.C., Rizal F.M., Lin T.H. Co-firing of fast pyrolysis bio-oil and heavy fuel oil in a 300-kWth furnace, Switzerland. Applied Sciences, 2016, vol. 6, no. 11, pp. 326.1-11.

22. Zabelkin S.A., Grachev A.N., Bashkirov V.N., Mullamukhametov F.I Energy application of pyrolysis liquid. Bulletin of the Technological University, 2010, no. 10, pp. 369-374. In Rus.

23. Loganathan K., Manoharan C. Evaluation of performance and emission features of jatropha biodiesel-turpentine blend as green fuel. Thermal Science, 2017, vol. 21, no. 1, pp. 615-625.

24. Nyashina G.S., Vershinina K.Y., Strizhak P.A. Impact of microexplosive atomization of fuel droplets on relative performance indicators of their combustion. Fuel Processing Technology, 2020, vol. 201, no. October, pp. 106334.1-14

25. Tuntsev D.V., Safin R.G., Kasimov A.M., Khismatov R.G., Sattarova Z.G. Technological scheme of gasification of a liquid product of contact pyrolysis. Bulletin of the Technological University, 2013, vol. 16, no. 19, pp. 139-141. In Rus.

26. Fortum. 2021. Available at: https://www.fortum.ru (assessed 11 November 2021).

27. Sippula O., Huttunen K., Hokkinen J., Kärki S., Suhonen H., Kajolinna T., Kortelainen M., Karhunen T., Jalava P., Uski O., YliPirilä P., Hirvonen M.R., Jokiniemi J. Emissions from a fastpyrolysis bio-oil fired boiler: comparison of health-related characteristics of emissions from bio-oil, fossil oil and wood. Environmental Pollution, 2019, vol. 248, pp. 888-897.

28. Ensyn Inc. 2021. Available at: http://www.ensyn.org/en/home (accessed 11 November 2021)

29. Valmet. 2021. Available at: https://www.valmet.com/ru/ (accessed 11 November 2021).

30. Stork Inc. 2021. Available at: https://www.stork.com/en (accessed 11 November 2021).
31. Vershinina K.Y., Shlegel N.E., Strizhak P.A. Impact of environmentally attractive additives on the ignition delay times of slurry fuels: experimental study. Fuel, 2019, vol. 238, pp. 275-288.

32. Zabelkin S.A., Tuntsev D.V., Grachev A.N., Bashkirov V.N. Energeticheskoe ispolzovanie zhidkikh produktov bystrogo piroliza drevesiny [Energy application of liquid products of fast pyrolysis of wood]. Bioenergetika i biotekhnologii, 2010, vol. 4, pp. 79-84.

33. Lupandin V., Thamburaj R., Nikolayev A. Test results of the OGT2500 gas turbine engine running on alternative fuels: BioOil, Ethanol, Biodiesel and Crude Oil. ASME Turbo Expo 2005: Power for Land, Sea, and Air. Reno, Nevada, International Gas Turbine Institute Publ., 2005. Vol. 1, pp. 421-426.

34. Opra Turbines Inc. 2021. Available at: https://www.opraturbines.com/ru/ (accessed 11 November 2021).

35. Van Beurden E., Pozarlik A., Putra B., Brem G., Bouten T., Withag J., Axelsson L.U. Improved CFD predictions of pyrolysis oil combustion using advanced spray measurements and numerical models. ASME Turbo Expo 2021: Turbomachinery Technical Conference and Exposition. Phoenix, International Gas Turbine Institute Publ., 2021. Vol. 6, pp. 1-12.

36. Bouten T., Withag J., Axelsson L., Putra B., Pozarlik A., Brem G., Benesch C., Brunner T ., Obernberger I. Experimental and numerical investigation of the application of fast pyrolysis oil in a gas turbine combustor. European Biomass Conference and Exhibition Proceedings. Twente, ETA-Florence Renewable Energies, 2020. pp. 957-965.

37. Seljak T., Širok B., Katrašnik T. Advanced fuels for gas turbines: fuel system corrosion, hot path deposit formation and emissions. Energy Conversion and Management, 2016, vol. 125, pp. 40-50.

38. Chiaramonti D., Oasmaa A., Solantausta Y. Power generation using fast pyrolysis liquids from biomass. Renewable and Sustainable Energy Reviews, 2007, vol. 11, no. 6, pp. 1056-1086.

39. VTT Technical Research Center of Finland Ltd. 2021. Available at: https://www.vttresearch.com/en/html (accessed 11 November 2021).

40. Wärtsilä Corp. 2021. Available at: https://www.wartsila.com/html (accessed 11 November 2021).

41. Solantausta Y., Nylund N.O., Gust S. Use of pyrolysis oil in a test diesel engine to study the feasibility of a diesel power plant concept. Biomass and Bioenergy, 1994, vol. 7, no. 1-6, pp. 297-306.

42. Yang S.I., Wu M.S. The droplet combustion and thermal characteristics of pinewood bio-oil from slow pyrolysis. Energy, 2017, vol. 141, pp. 2377-2386

43. Kim T.Y., Lee S., Kang K. Performance and emission characteristics of a high-compression-ratio diesel engine fueled with wood pyrolysis oil-butanol blended fuels. Energy, 2015, vol. 93, pp. 2241-2250.

44. Lee S., Kim T.Y., Kang K., Park I., Kim M., Lim J. Performance and emission charactertistics of an IDI diesel generator fueled with wood pyrolysis oil-butanol blended fuels. Transactions of the Korean Society of Automotive Engineers, 2017, vol. 25, no. 3, pp. 380-388.

45. Lee S., Kim Y., Kang K. Combustion and emission characteristics of wood pyrolysis oil and N-Butanol-Blended fuel in a diesel engine. The Role of Exergy in Energy and the Environment. London, Springer International Publ., 2018. pp. 171-187.

46. Lee S., Kim T.Y. Feasibility study of using wood pyrolysis oilethanol blended fuel with diesel pilot injection in a diesel engine. Fuel, 2015, vol. 162, pp. 65-73.

47. Likhanov V.A., Kozlov A.N., Araslanov M.I. Effect of ethanol and rape oil application on the indicators of the combustion process and sages containment in the tractor diesel cylinder $2 \mathrm{Ch}$ 10.5/12.0 depending on load change. Vestnik chuvashskoy gosudarstvennoy sel'skokhozyaystvennoy akademii, 2018, vol. 4, no. 1, pp. 59-64. In Rus.

48. BTG Biomass Technology Group. 2021. Available at: https://www.btgworld.com/html (accessed 11 November 2021).

49. Dunaev S.V., Isaev A.V., Lesin A.V., Popov V.P., Fedin K.I. Problems of the increasing use of natural gas as a motor fuel. World of oil products. Bulletin of oil companies, 2017, no. 1, pp. 10-14. In Rus.

50. Chirkov V.G. Mini thermal power stations operating on pyrolyzed fuel. Thermal Engineering, 2007, no. 8, pp. 35-39. In Rus.

51. Tuntsev D., Khismatov R., Kasimov A., Romancheva I., Savelev A. Rezultaty eksperementalnogo issledovaniya protsessa gazifikatsii 
zhidkogo produkta bystrogo piroliza drevesnykh otkhodov lesmnoy promyshlennosti [Results of experimental studies of the gasification process liquid products fast pyrolysis of waste wood for the wood industry]. Aktualnye napravleniya nauchnykh issledovany XXI veka: teoriya i praktika [Actual directions of scientific researches of the XXI century: theory and practice]. Voronezh State University of Forestry and Technologies named after G.F. Morozov, 2016. Vol. 2, no. 5, pp. 352-356.

52. Abou Rjeily M., Gennequin C., Pron H., Abi-Aad E., Randrianalisoa J.H. Pyrolysis-catalytic upgrading of bio-oil and pyrolysiscatalytic steam reforming of biogas: a review. Environmental Chemistry Letters, 2021, vol. 19, no. 4, pp. 2825-2872.

53. You S., Ok Y.S., Tsang D.C.W., Kwon E.E., Wang C.H. Towards practical application of gasification: a critical review from syngas and biochar perspectives. Critical Reviews in Environmental Science and Technology, 2018, vol. 48, no. 22-24, pp. 1165-1213.

54. Baba Y., Pandyaswargo A.H., Onoda H. An analysis of the current status of woody biomass gasification power generation in japan. Energies, 2020, vol. 13, no. 18, pp. 4903.1-13.

55. Calì G., Deiana P., Bassano C., Meloni S., Maggio E., Mascia M., Pettinau A. Syngas production, clean-up and wastewater management in a demo-scale fixed-bed updraft biomass gasification unit. Energies, 2020, vol. 13, no. 10, pp. 2594.1-15.

56. Siemens AG. 2004-2021. Available at: https://new.siemens.com/ $\mathrm{ru} / \mathrm{ru} . \mathrm{html}$ (accessed 11 November 2021).

57. Ahrenfeldt J., Thomsen T.P., Henriksen U., Clausen L.R. Biomass gasification cogeneration - A review of state of the art technology and near future perspectives. Applied Thermal Engineering, 2013, vol. 50, no. 2, pp. 1407-1417.

58. Kasimov A.M. Sovershenstvovanie tekhnologii i oborudovaniva termokhimicheskoy pererebotki drevesnykh otkhodov v vysokokaloriyny generatorny gaz. Avtoreferat Dis. Kand. nauk [Improvement of technology and equipment for thermochemical processing of wood waste into high-calorie generator gas. Cand. Diss. Abstract]. Kazan, 2017. 16 p.

59. Fayzrakhmanova G.M. Modifikatsiya bitumnykh vyazhushchikh zhidkimi produktami bystrogo ablyatsionnogo piroliza drevesiny. Avtoreferat Dis. Kand. nauk [Modification of bituminous binders with liquid products of rapid ablative pyrolysis of wood. Cand. Diss. Abstract]. Kazan, 2015. 16 p.

60. Fayzrakhmanova G.M., Zabelkin S.A., Grachev A.N., Bashkirov V.N Development of technology of production of organic binder for road construction using products of thermal processing of wood biomass. Bulletin of the Amur State University, 2015, vol. 19, no. 2, pp. 79-85. In Rus.

61. Kiselev V.P. Kompleksnoe ispolzovanie otkhodov khimicheskoy pererabotki biomassy dereva i drugikh vtorichnykh resursov v proizvodstve kompozitsionnykh vyazhushchikh $i$ materialov, poluchennykh na ikh osnove. Avtoreferat Dis. Dokt. nauk [Complex use of wastes from chemical processing of wood biomass and other secondary resources in the production of composite binders and materials obtained on their basis. Dr. Diss. Abstract]. Krasnoyarsk, 2006. $43 \mathrm{p}$.

62. Zabelkin S., Bikbulatova G., Grachev A., Bashkirov V., Burenkov S., Makarov A. Modification of bitumen binder by the liquid products of wood fast pyrolysis. Road Mater. Pavement Des., 2019, vol. 20, no. 5 , pp. 1182-1200.

63. Wang H., Ma Z., Chen X., Mohd Hasan M.R. Preparation process of bio-oil and bio-asphalt, their performance, and the application of bio-asphalt: a comprehensive review. Journal of Traffic and Transportation Engineering (English Edition). Periodical Offices of Changan University, 2020, vol. 7, no. 2, pp. 137-151.

64. Barbero-López A., Akkanen J., Lappalainen R., Peräniemi S., Haapala A. Bio-based wood preservatives: Their efficiency, leaching and ecotoxicity compared to a commercial wood preservative. Sci. Total Environ., 2021, vol. 753, pp. 142013.1-7.

65. Mazela B. Fungicidal value of wood tar from pyrolysis of treated wood. Waste Manag., 2007, vol. 27, no. 4, pp. 461-465.

66. Meier D., Andersons B., Irbe I., Chirkova J., Faix O. Preliminary study on fungicide and sorption effects of fast pyrolysis liquids used as wood preservative. Prog. Thermochem. Biomass Convers. 2001, pp. 1550-1563.

67. Kim K.H., Jeong H.S., Kim J.-Y., Han G.S., Choi I.-G., Choi J.W. Evaluation of the antifungal effects of bio-oil prepared with ligno- cellulosic biomass using fast pyrolysis technology. Chemosphere, 2012, vol. 89, no. 6, pp. 688-693.

68. Temiz A., Akbas S., Panov D., Terziev N., Alma M.H., Parlak S., Kose G. Chemical composition and efficiency of bio-oil obtained from giant cane (Arundo donax L.) as a wood preservative. BioResources, 2013, vol. 8, no. 2, pp. 2084-2098.

69. Hassan E.B., El-Giar E.M., Steele P. Evaluation of the antioxidant activities of different bio-oils and their phenolic distilled fractions for wood preservation. Int. Biodeterior. Biodegrad., vol. 110, pp. $121-128$.

70. Ehrbe S.V. Neftyanaya antisepticheskaya zhidkost dlya propitki drevesiny [Petroleum antiseptic liquid for impregnation of timber]. Patent RF, no. 2455154C1, 2012.

71. Ma Y., Gong X., Wang C. Research Progress in Wood Adhesives. Chemistry and Industry of Forest Products, 2020, vol. 40, no. 2, pp. 1-15.

72. Valeeva A.R., Grachev A.N., Zabelkin S.A., Bashkirov V.N., Sabirzyanova A.I. The strength of the modified phenol formaldehyde resin, depending on the degree of additional of wood pyrolysis liquid. Derevoobrobativaushaya promishlenoost, 2020, no. 1, pp. 88-95. In Rus.

73. Zabelkin S.A., Grachev A.N., Bashkirov V.N., Cherezova E.N. Modification of phenol-formaldehyde resins with liquid products of wood pyrolysis and study of their gluing ability. Bulletin of the Technological University, 2010, vol. 8, pp. 440-444. In Rus.

74. Zabelkin S.A., Grachev A.N., Bashkirov V.N., Cherezova E.N. Modification of phenol-formaldehyde resins with liquid products of wood pyrolysis. Bulletin of the Technological University, 2014, vol. 17, no. 10, pp. 97-100. In Rus.

75 Mao A., Shi S.Q., Steele P. Flakeboard bonded with polymeric diphenylmethane diisocyanate/bio-oil adhesive systems. Forest Products Journal, 2011, vol. 61, no. 3, pp. 240-245.

76. Jia L., Chu J., Ma L., Qi X., Kumar A. Life cycle assessment of plywood manufacturing process in China. International Journal of Environmental Research and Public Health, 2019, vol. 16, no. 11, pp. 2037.1-10

77. Ren X., Cai H., Du H., Chang J. The preparation and characterization of pyrolysis bio-oil-resorcinol-aldehyde resin cold-set adhesives for wood construction. Polymers, 2017, vol. 9, no. 6, pp. 232.1-11.

78. Ayrilmis N., Özbay G. Technological properties of plywood bonded with phenol-formaldehyde resol resin synthesized with bio-oil. Cerne, 2017, vol. 23, no. 4, pp. 493-500.

79. Mao A., Xu W., Xi E., Li Q., Wan H. Evaluation of phenolformaldehyde resins modified and blended with pyrolysis bio-oil for plywood. Forest Products Journal, 2018, vol. 68, no. 2, pp. 113-119.

80. Omar N.N., Mustafa I.S., Abdullah N., Hashim R. The use of environmentally friendly bio-oil in the production of phenol formaldehyde (PF) resin. Pertanika Journal of Science and Technology, 2018, vol. 26, no. 1, pp. 177-192.

81. Adhikari S., Auad M., Via B., Shah A., Patil V. Production of novolac resin after partial substitution of phenol from bio-oil. Transactions of the ASABE, 2020, vol. 63, no. 4, pp. 901-912.

82. Dong F., Wang M., Wang Z. Bio-oil as substitute of phenol for synthesis of resol-type phenolic resin as wood adhesive. International Journal of Chemical Reactor Engineering, 2018, vol. 16, no. 3, pp. 20170107.1-8.

83 Liu Y., Via B.K., Pan Y., Cheng Q., Guo H., Auad M.L., Taylor S. Preparation and characterization of epoxy resin cross-linked with high wood pyrolysis bio-oil substitution by acetone pretreatment. Polymers, 2017, vol. 9, no. 3, pp. 106.1-14.

84. Zabelkin S.A., Grachev A.N., Bikbulatova G.M., Yakovleva A.E., Makarov A.A., Bashkirov V.N. Resole phenol-formaldehyde resin with addition of neutralized liquid products of birch fast pyrolysis. Klei. Germetiki. Tekhnologii, 2017, no. 10, pp. 19-23. In Rus.

85. Patel M., Oyedun A.O., Kumar A., Gupta R. A techno-economic assessment of renewable diesel and gasoline production from aspen hardwood. Waste and Biomass Valorization, 2019, vol. 10, no. 10 , pp. $2745-2760$

86. Pisupati S.V., Bhalla S. Influence of calcium content of biomassbased materials on simultaneous NOx and $\mathrm{SO} 2$ reduction. Environmental Science and Technology, 2008, vol. 42, no. 7, pp. 2509-2514. 
87. Pisupati S.V., Bhalla S. Numerical modeling of NOx reduction using pyrolysis products from biomass-based materials. Biomass and Bioenergy, 2008, vol. 32, no. 2, pp. 146-154.

88. Simons G.A., Place W.J., Oehr K.H., Zhou J., Pisupati S.V., Wojtowicz M.A., Bassilakis R. Simultaneous $\mathrm{SO}_{2} / \mathrm{NO} / \mathrm{N}_{2} \mathrm{O}$ control in coal fired systems using BioLimeTM. Proceedings of the International Technical Conference on Coal Utilization \& Fuel Systems. Coal \& Slurry Technology Assoc., 1997, no. 3, pp. 739-749.

89. Vélez D.C.P., Magalhães W.L.E., Capobianco G. Carbon fiber from fast pyrolysis bio-oil. Science and Technology of Materials, 2018, vol. 30, pp. 16-22.

90. Xin X., Dell K., Udugama I.A., Young B.R., Baroutian S. Transforming biomass pyrolysis technologies to produce liquid smoke food flavouring. Journal of Cleaner Production, 2021, vol. 294, pp. 125368.1-37.

91. Onofreychuk A.O. Fractional distillation of pyrolysis distillate. Bulatovskie chteniya, 2018, no. 5, pp. 238-240. In Rus.

92. Jacobson K., Maheria K.C., Kumar Dalai A. Bio-oil valorization: a review. Renewable and Sustainable Energy Reviews, 2013, vol. 23, pp. 91-106.

93. GOST R 56861-2016. Sistema upravleniya zhiznennym tsiklom. Razrabotka kontseptsii izdeliya i tekhnologiy. Obshchie polozheniya [State Standard 56861-2016. Life cycle management system. Product conception and technologies development. General provisions]. Moscow, StandardInform Publ., 2019. 10 p.

Received: 1 December 2021.

\section{Information about the authors}

Konstantin V. Slyusarsky, Cand. Sc., associate professor, National Research Tomsk Polytechnic University.

Kirill B. Larionov, Cand. Sc., head of the laboratory, T.F. Gorbachev Kuzbass State Technical University.

Elena N. Ivashkina, Dr. Sc., professor, National Research Tomsk Polytechnic University.

Alexander S. Zavorin, Dr. Sc., head of the department, National Research Tomsk Polytechnic University.

Vladimir E. Gubin, Cand. Sc., associate professor, National Research Tomsk Polytechnic University. 